\title{
Synthesis, characterization and evaluation of 1,3,5-triazine aminobenzoic acid derivatives for their antimicrobial activity
}

\author{
Khadijah M. Al-Zaydi ${ }^{*}$, Hosam H. Khalil2, Ayman El-Faham² and Sherine N. Khattab²*
}

\begin{abstract}
Background: Replacement of chloride ions in cyanuric chloride give several variants of 1,3,5-triazine derivatives which were investigated as biologically active small molecules. These compounds exhibit antimalarial, antimicrobial, anti-cancer and anti-viral activities, among other beneficial properties. On the other hand, treatment of bacterial infections remains a challenging therapeutic problem because of the emerging infectious diseases and the increasing number of multidrug-resistant microbial pathogens. As multidrug-resistant bacterial strains proliferate, the necessity for effective therapy has stimulated research into the design and synthesis of novel antimicrobial molecules.

Results: 1,3,5-Triazine 4-aminobenzoic acid derivatives were prepared by conventional method or by using microwave irradiation. Using microwave irradiation gave the desired products in less time, good yield and higher purity. Esterification of the 4-aminobenzoic acid moiety afforded methyl ester analogues. The s-triazine derivatives and their methyl ester analogues were fully characterized by FT-IR, NMR ( ${ }^{1} \mathrm{H}-\mathrm{NMR}$ and $\left.{ }^{13} \mathrm{C}-\mathrm{NMR}\right)$, mass spectra and elemental analysis. All the synthesized compounds were evaluated for their antimicrobial activity. Some tested compounds showed promising activity against Staphylococcus aureus and Escherichia coli.

Conclusions: Three series of mono-, di- and trisubstituted s-triazine derivatives and their methyl ester analogues were synthesized and fully characterized. All the synthesized compounds were evaluated for their antimicrobial activity. Compounds (10), (16), (25) and (30) have antimicrobial activity against $S$. aureus comparable to that of ampicillin, while the activity of compound (13) is about 50\% of that of ampicillin. Compounds (13) and (14) have antimicrobial activity against $E$. coli comparable to that of ampicillin, while the activity of compounds (9-12) and (15) is about 50\% of that of ampicillin. Furthermore, minimum inhibitory concentrations values for clinical isolates of compounds (10), (13), (14), (16), (25) and (30) were measured. Compounds (10) and (13) were more active against MRSA and E. coli than ampicillin. Invitro cytotoxicity results revealed that compounds (10) and (13) were nontoxic up to $250 \mu \mathrm{g} / \mathrm{mL}$ (with $\mathrm{SI}=10$ ) and $125 \mu \mathrm{g} / \mathrm{mL}$ (with $\mathrm{SI}=5$ ), respectively.
\end{abstract}

Keywords: 1,3,5-Triazine derivatives, 4-Aminobenzoic acid, Morpholine, Piperidine, Aniline, Benzylamine, Diethylamine, Microwave irradiation, Antimicrobial activity

\section{Background}

Sophisticated s-triazine derivatives can be easily prepared from the cheap and readily available

\footnotetext{
*Correspondence: kalzaydi@kau.edu.sa; sh.n.khattab@gmail.com ${ }^{1}$ Department of Chemistry, Faculty of Sciences-AL Faisaliah, King Abdulaziz University, Jeddah, P.O. Box 50918, Jeddah 21533, Kingdom of Saudi Arabia

2 Department of Chemistry, Faculty of Science, Alexandria University, P.O. Box 426, Ibrahimia, Alexandria 21321, Egypt
}

2,4,6-trichloro-1,3,5-triazine (cyanuric chloride) 1 [13]. Replacement of chloride ions in cyanuric chloride give several variants of 1,3,5-triazine derivatives, which were investigated as biologically active small molecules [4-8]. These compounds exhibit antimalarial [9-16], antimicrobial [17-25], anti-cancer [26-31] and antiviral activities [32], among other beneficial properties. On the other hand, treatment of bacterial infections remains a challenging therapeutic problem because of 
emerging infectious diseases and the increasing number of multidrug-resistant microbial pathogens [33]. As multidrug-resistant bacterial strains proliferate, the necessity for effective therapy has stimulated research into the design and synthesis of novel antimicrobial molecules [34-42].

In this study, we prepared several 1,3,5-triazine derivatives by replacing one, two, or three chloride ions of cyanuric chloride with different $N$-nucleophiles (including 4-aminobenzoic acid, methyl p-aminobenzoate, aniline, benzylamine, diethylamine, morpholine and piperidine) and evaluated their antimicrobial activity.

\section{Results and discussion Chemistry}

Cyanuric chloride (1) is definitely an excellent starting compound for the straight forward preparation of highly structured multitopic molecules [1]. The first substitution is exothermic; therefore, the temperature of the reaction mixture has to be maintained at $0{ }^{\circ} \mathrm{C}$. The substitution of the second chloride can be performed at room temperature. Finally, the third position is functionalized under reflux of the solvent. As a result, a careful control of the reaction temperature during the substitution reactions will allow the synthesis of 2,4,6-trisubstituted-1,3,5triazines by the sequential and very selective addition of amine nucleophiles [1, 43].
A monosubstituted 1,3,5-triazine series was prepared selectively by substituting one chloride ion of cyanuric chloride (1) with 4-aminobenzoic acid (2), aniline, benzylamine, diethylamine, morpholine or piperidine in the presence of sodium carbonate as an acid scavenger of the liberated hydrochloric acid, in an ice-bath, to afford products 4-((4,6-dichloro-1,3,5-triazin-2yl)amino) benzoic acid (3), 4,6-dichloro- $N$-phenyl-1,3,5-triazin2-amine (4), $N$-benzyl-4,6-dichloro-1,3,5-triazin-2-amine (5), 4,6-dichloro- $N, N$-diethyl-1,3,5-triazin-2-amine (6), 4-(4,6-dichloro-1,3,5-triazin-2-yl)morpholine (7), and 2,4-dichloro-6-(piperidin-1-yl)-1,3,5-triazine (8), respectively (Scheme 1). The structure of the products was confirmed using NMR $\left({ }^{1} \mathrm{H}\right.$ and $\left.{ }^{13} \mathrm{C}\right)$, IR and elemental analysis.

In addition, a series of disubstituted 1,3,5-triazine was prepared by replacement of the second chloride ion of 4-((4,6-dichloro-1,3,5-triazin-2-yl)amino)benzoic acid (3) with aniline, benzylamine, diethylamine, morpholine, or piperidine, respectively. The reaction mixture was stirred overnight at room temperature in presence of sodium carbonate to give 4-((4-chloro-6-(phenylamino)-1,3,5triazin-2-yl)amino)benzoic acid (9), 4-((4-(benzylamino)6-chloro-1,3,5-triazin-2-yl)amino)benzoic acid (10), 4-((4-chloro-6-(diethylamino)-1,3,5-triazin-2-yl)amino) benzoic acid (11), 4-((4-chloro-6-morpholino-1,3,5-triazin-2-yl)amino)benzoic acid (12), and 4-((4-chloro-6(piperidin-1-yl)-1,3,5-triazin-2-yl)amino)benzoic acid (13),

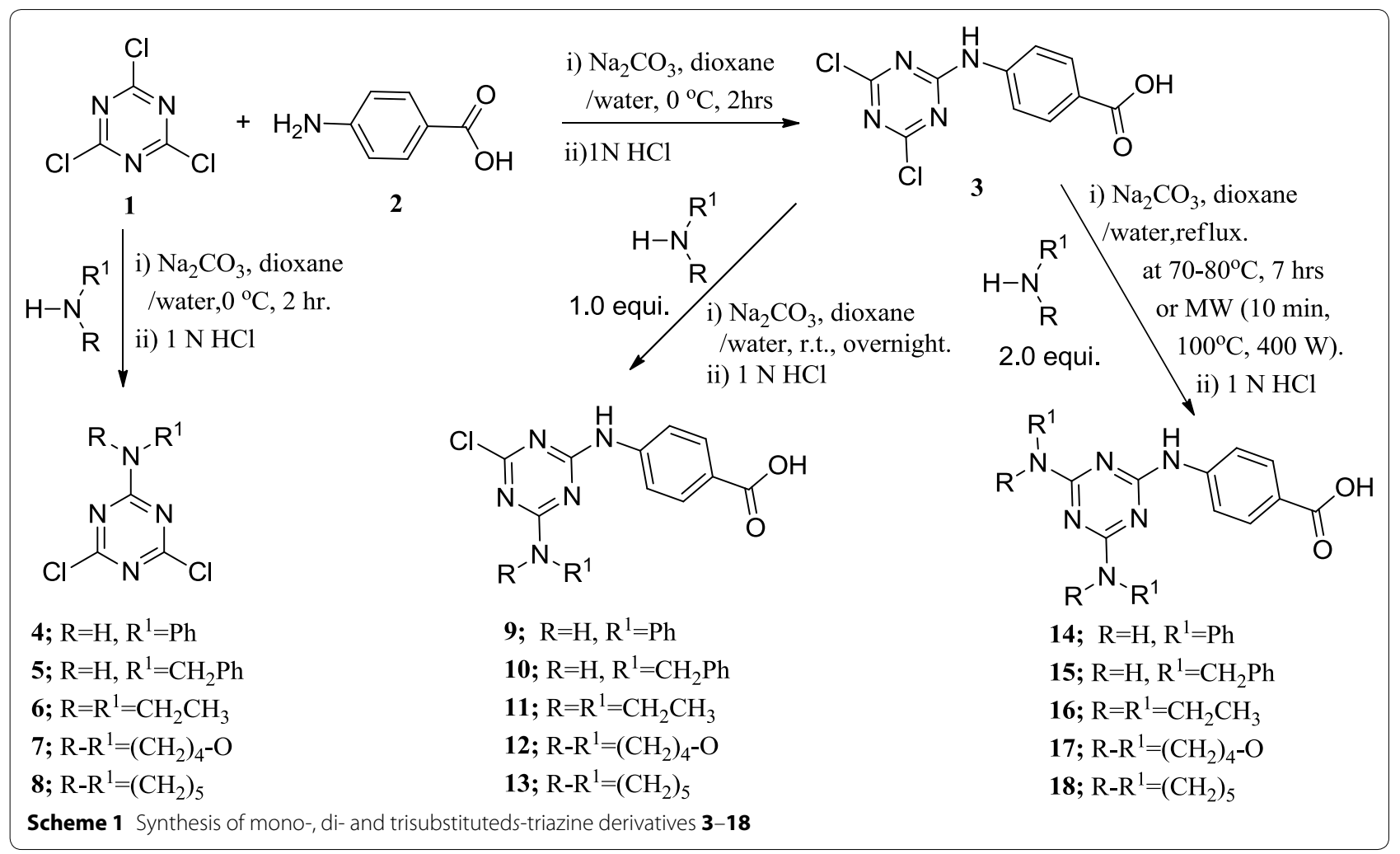


respectively (Scheme 1 ). The structure of the products was confirmed using NMR $\left({ }^{1} \mathrm{H}\right.$ and $\left.{ }^{13} \mathrm{C}\right)$, IR, mass spectra and elemental analysis.

The ${ }^{1} \mathrm{H}-\mathrm{NMR}$ of compound (11) in DMSO- $d_{6}$ showed that the two ethyl groups of the diethylamino moiety are not equivalent, as indicated from their chemical shift values, where the two methyl groups are observed at 1.09 and $1.15 \mathrm{ppm}$ as two triplets and the methylene groups are observed at the range $3.50-3.53 \mathrm{ppm}$ as a multiplet. These observations indicate that the two ethyl groups are found in different electronic environments. This fact can be attributed to restricted rotation around the $\mathrm{C}_{6}-\mathrm{N}$ bond due to resonance that gives this bond some double bond character [44]. The difference in the chemical shifts of ethyl groups are probably due to differences in the field effects (anisotropic effects) of the benzoyl group of 4-aminobenzoic acid substituents on $\mathrm{C}_{2}$ on either ethyl groups (Fig. 1). The aromatic protons appear as two doublets at chemical shifts 7.79 and $7.86 \mathrm{ppm}$. The two $\mathrm{D}_{2} \mathrm{O}$ exchangeable protons ( $\mathrm{NH}$ and $\mathrm{OH}$ ) are observed at 10.34 and $12.69 \mathrm{ppm}$, respectively. The previous prediction was confirmed by the ${ }^{13} \mathrm{C}$-NMR spectrum of compound (11), where the two methyl carbons appear as two peaks at 13.01 and $13.47 \mathrm{ppm}$ and the two methylene carbons are observed at 42.01 and $42.47 \mathrm{ppm}$.

Furthermore, $\mathrm{C}_{2}$-symmetrical 1,3,5-triazine tripod series were prepared by replacing the two chloride ions of 4-((4,6-dichloro-1,3,5-triazin-2-yl)amino)benzoic acid (3) with two equivalents of aniline, benzylamine, diethylamine, morpholine, or piperidine, respectively. The reaction proceeded at $70-80^{\circ} \mathrm{C}$ in dioxane/water solvent mixture using sodium carbonate as a base by the conventional method; or by using microwave irradiation and employing a multimode reactor (Synthos 3000, Aton Paar $\mathrm{GmbH}, 1400 \mathrm{~W}$ maximum magnetron) to produce the corresponding products: 4-((4,6-bis(phenylamino)-1,3,5triazin-2-yl)amino)benzoicacid(14),4-((4,6-bis(benzylamino)1,3,5-triazin-2-yl)amino)benzoic acid (15), 4-((4,6bis(diethylamino)-1,3,5-triazin-2-yl)amino)benzoic acid (16), 4-((4,6-dimorpholino-1,3,5-triazin-2-yl)amino) benzoic acid (17), 4-((4,6-di(piperidin-1-yl)-1,3,5triazin-2-yl)amino)benzoic acid (18), respectively

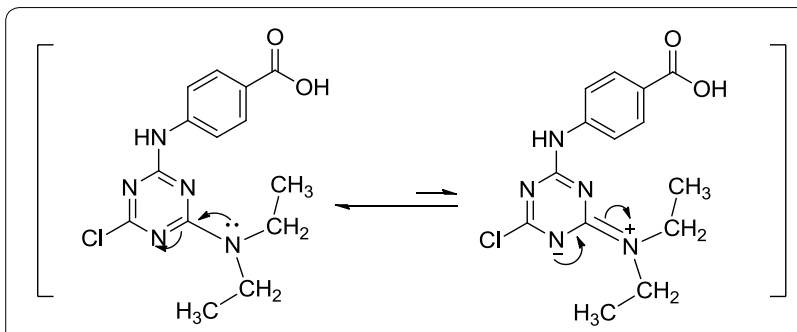

Fig. 1 Restricted rotation around $\mathrm{C}_{6}-\mathrm{N}$ bond due to resonance
(Scheme 1). Using microwave irradiation produced the desired products in less time, good yield and higher purity. The structures of the products were confirmed using NMR $\left({ }^{1} \mathrm{H}\right.$ and $\left.{ }^{13} \mathrm{C}\right)$, IR, mass spectra and elemental analysis. As a prototype of this series, in the ${ }^{1} \mathrm{H}-\mathrm{NMR}$ of compound 17, the methylene group protons of morpholine moiety appear as multiplet at the chemical shift range of 3.60-3.69 ppm, and the $p$-disubstituted benzene ring protons appear as two doublets at chemical shifts 7.73 and $7.83 \mathrm{ppm}$. The $\mathrm{N}-\mathrm{H}$ proton appears at the chemical shift range of $9.74-9.87 \mathrm{ppm}$ as a broad $\mathrm{D}_{2} \mathrm{O}$ exchangeable peak.

Similarly, another tripod series of 1,3,5-triazine derivatives (19-22) was synthesized, which differed only in one position of the triazine nucleus, but contained 4-aminobenzoic acid and morpholino moieties in all members of this series. Chloride ion of compounds (9-11) and (13) was replaced with one equivalent of morpholine at $70-80{ }^{\circ} \mathrm{C}$ in a dioxane/water solvent mixture using sodium carbonate as a base by the conventional method, or by using microwave irradiation, employing a multimode reactor (Synthos 3000, Aton Paar GmbH, 1400 W maximum magnetron) to give the corresponding products (Scheme 2). Using microwave irradiation produced the desired products in less time, good yield and higher purity. The structures of the products were confirmed using NMR $\left({ }^{1} \mathrm{H}\right.$ and $\left.{ }^{13} \mathrm{C}\right)$, IR and elemental analyses. As a prototype of this series, in the ${ }^{1} \mathrm{H}-\mathrm{NMR}$ of compound (20), the eight methylene protons of morpholine appear as multiplet at a chemical shift range of 3.57-3.74 ppm, while the benzyl methylene group protons appear as multiplet at $4.45-4.50 \mathrm{ppm}$. The nine aromatic protons appear as multiplet at chemical shift ranges of 7.18-7.71 and 7.69-7.85 ppm. The $\mathrm{N}-\mathrm{H}$ proton $\left(\mathrm{D}_{2} \mathrm{O}\right.$ exchangeable) appears as multiplet at 9.49-9.73 ppm due to its coupling with the adjacent methylene protons. The $\mathrm{O}-\mathrm{H}$ proton $\left(\mathrm{D}_{2} \mathrm{O}\right.$ exchangeable) is observed at $12.40 \mathrm{ppm}$ as a singlet peak. The multiplet appearance of benzyl methylene protons indicates that the two protons are not equivalent (enantiotopic protons). From the conformational point of view, using the Newman projection formula (Fig. 2), $\mathrm{H}_{\mathrm{a}}$ and $\mathrm{H}_{\mathrm{b}}$ are not equivalent due to restricted rotation around the $\mathrm{C}_{4}-\mathrm{N}$ bond (Fig. 1); as a result, they can couple via germinal coupling depending on the $\mathrm{H}_{\mathrm{a}} \mathrm{CH}_{\mathrm{b}}$ angle in addition to their vicinal coupling of the $\mathrm{NH}_{\mathrm{c}}$ proton, as shown by the staggered conformation of (20) (Fig. 2). This behavior also explains the multiplet appearance of the $\mathrm{N}-\mathrm{H}_{\mathrm{c}}$ proton.

Furthermore, esterification of the previously prepared compounds (14-22) afforded compounds (23-31) (Scheme 3). The structure of the products was confirmed using NMR $\left({ }^{1} \mathrm{H}\right.$ and $\left.{ }^{13} \mathrm{C}\right)$, IR, mass spectra and elemental analysis. 
<smiles>[R]N([R])c1nc(Cl)nc(Nc2ccc(C(=O)O)cc2)n1</smiles>

9; $\mathrm{R}=\mathrm{H}, \mathrm{R}^{1}=\mathrm{Ph}$.

10; $\mathrm{R}=\mathrm{H}, \mathrm{R}^{1}=\mathrm{CH}_{2} \mathrm{Ph}$.

11; $\mathrm{R}=\mathrm{R}^{1}=\mathrm{CH}_{2} \mathrm{CH}_{3}$.

13; $\mathrm{R}-\mathrm{R}^{1}=\left(\mathrm{CH}_{2}\right)_{5}$.

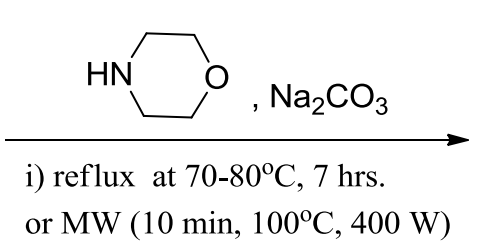

ii) $1 \mathrm{~N} \mathrm{HCl}$<smiles>[R]N([R])c1nc(Nc2ccc(C(=O)O)cc2)nc(N2CCOCC2)n1</smiles>

19; $\mathrm{R}=\mathrm{H}, \mathrm{R}^{1}=\mathrm{Ph}$.

20; $\mathrm{R}=\mathrm{H}, \mathrm{R}^{1}=\mathrm{CH}_{2} \mathrm{Ph}$.

21; $\mathrm{R}=\mathrm{R}^{1}=\mathrm{CH}_{2} \mathrm{CH}_{3}$.

22; $\mathrm{R}-\mathrm{R}^{1}=\left(\mathrm{CH}_{2}\right)_{5}$.

Scheme 2 Synthesis of tripod morpholino-s-triazine derivatives 19-22

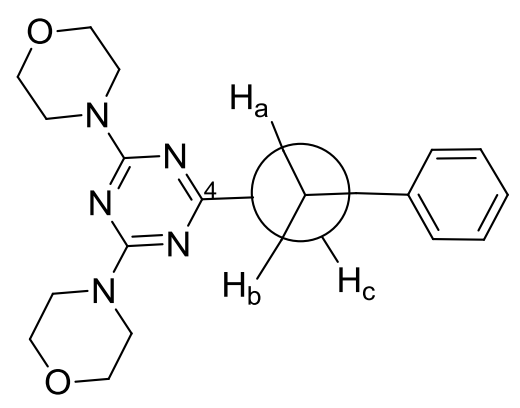

Fig. 2 Newman projection formula of $\mathrm{N}$-(4-benzylamino-6-morpholino-1,3,5-triazin-2-yl) aminobenzoic acid 20

\section{Antimicrobial activity}

The synthesized compounds (9-31) have been evaluated for their antimicrobial activity against E. coli representing Gram-negative bacteria, S. aureus representing Gram-positive bacteria and $C$. albicans representing fungi. Microdilution susceptibility test in Müller-Hinton Broth (Oxoid) and Sabouraud Liquid Medium (Oxoid) were used for the determination of antibacterial and antifungal activity [45]. The minimal inhibitory concentration (MIC) values listed in Table 1 showed that all tested compounds have lower antifungal activity than clotrimazole (Canesten ${ }^{\circledR}$, Bayer).

The synthesized compounds are more active against $S$. aureus and E. coli. Compounds (10), (16), (25) and (30) have antimicrobial activity against $S$. aureus comparable to that of ampicillin, while the activity of compound (13) is about $50 \%$ of that of ampicillin. Compounds (13) and (14) have antimicrobial activity against E. coli comparable to that of ampicillin, while the activity of compounds (9-12) and (15) is about 50\% of that of ampicillin.

Furthermore, minimum inhibitory concentrations (MIC $\mu \mathrm{g} / \mathrm{mL}$ ) values for clinical isolates of compounds
(10), (13), (14), (16), (25) and (30) were also tested and listed in Table 2. Compounds (10) and (13) were more active against MRSA and $E$. coli than ampicillin.

Invitro cytotoxicity of the most active compounds (10) and (13) were carried out with 5ero cell line using Mosmann method with certain modifications as described in the literature [46]. 50\% cytotoxic concentration $\left(\mathrm{CC}_{50}\right)$ expressed in $\mu \mathrm{g} / \mathrm{mL}$ and selectivity index (SI) values were listed in Table 3 . The results revealed that the test compounds (10) and (13) were nontoxic up to $250 \mu \mathrm{g} / \mathrm{mL}$ (with $\mathrm{SI}=10$ ) and $125 \mu \mathrm{g} / \mathrm{mL}$ (with $\mathrm{SI}=5$ ), respectively.

\section{Experimental section Chemistry}

Solvents and reagents were purchased from SigmaAldrich. Unless otherwise stated, the normal workup from organic solvent involved drying over $\mathrm{Na}_{2} \mathrm{SO}_{4}$ and rotary evaporation. TLC was performed using aluminum-backed Merck Silica Gel 60 F-254 plates using suitable solvent systems with spots being visualized by a Spectroline UV Lamp ( 254 or $365 \mathrm{~nm}$ ) or $\mathrm{I}_{2}$ vapor. Melting points were obtained in open capillary tubes using a MEL-Temp II melting point apparatus and are uncorrected. Microwave experiments were performed using a multimode reactor (Synthos 3000, Aton Paar GmbH, $1400 \mathrm{~W}$ maximum magnetron). Infrared spectra (IR) were recorded on a Perkin-Elmer 1600 series Fourier transform instrument as $\mathrm{KBr}$ pellets. The absorption bands $(\max )$ are given in wave numbers $\left(\mathrm{cm}^{-1}\right)$. Nuclear magnetic resonance (NMR) spectra $\left({ }^{1} \mathrm{H}-\mathrm{NMR}\right.$ and ${ }^{13} \mathrm{C}$ NMR) were recorded on a JEOL $500 \mathrm{MHz}$ spectrometer at ambient temperature. Chemical shifts are reported in parts per million ( $\mathrm{ppm}$ ) and are referenced relative to residual solvent (e.g. $\mathrm{CHCl}_{3}$ at $\delta \mathrm{H} 7.26 \mathrm{ppm}$ for $\mathrm{CDCl}_{3}$, DMSO at $\delta \mathrm{H} 2.50 \mathrm{ppm}$ for DMSO- $\mathrm{d}_{6}$ ). Spin multiplicities are represented by the following signals: singlet (s), 


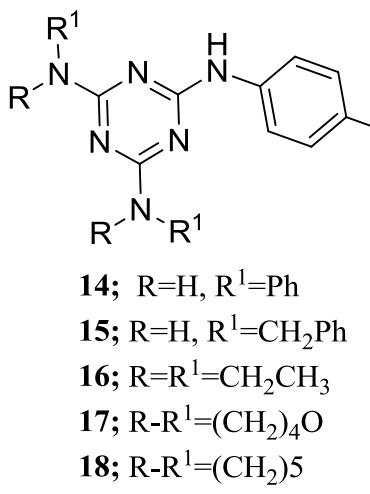<smiles>C1COCCN1</smiles><smiles>[R]N([R])c1ncnc(Nc2ccc(C(=O)O)cc2)n1</smiles>

19; $\mathrm{R}=\mathrm{H}, \mathrm{R}^{1}=\mathrm{Ph}$

20; $\mathrm{R}=\mathrm{H}, \mathrm{R}^{1}=\mathrm{CH}_{2} \mathrm{Ph}$

21; $\mathrm{R}=\mathrm{R}^{1}=\mathrm{CH}_{2} \mathrm{CH}_{3}$

22; $\mathrm{R}-\mathrm{R}^{1}=\left(\mathrm{CH}_{2}\right)_{5}$ i) $\mathrm{CH}_{3} \mathrm{OH} /$ conc. $\mathrm{H}_{2} \mathrm{SO}_{4}$

ii) $\mathrm{NaHCO}_{3}$ solution.<smiles>[R]N([R])c1nc(Nc2ccc(C(=O)OC)cc2)nc(N([R])[R])n1</smiles>

23; $\mathrm{R}=\mathrm{H}, \mathrm{R}^{1}=\mathrm{Ph}$

24; $\mathrm{R}=\mathrm{H}, \mathrm{R}^{1}=\mathrm{CH}_{2} \mathrm{Ph}$

25; $\mathrm{R}=\mathrm{R}^{1}=\mathrm{CH}_{2} \mathrm{CH}_{3}$

26; $\mathrm{R}-\mathrm{R}^{1}=\left(\mathrm{CH}_{2}\right)_{4} \mathrm{O}$

27; $\mathrm{R}-\mathrm{R}^{1}=\left(\mathrm{CH}_{2}\right) 5$

i) $\mathrm{CH}_{3} \mathrm{OH} /$ conc. $\mathrm{H}_{2} \mathrm{SO}_{4}$

ii) $\mathrm{NaHCO}_{3}$ solution.<smiles>[R]N([R])c1nc(Nc2ccc(C(=O)OC)cc2)nc(N2CCOCC2)n1</smiles>

28; $\mathrm{R}=\mathrm{H}, \mathrm{R}^{1}=\mathrm{Ph}$.

29; $\mathrm{R}=\mathrm{H}, \mathrm{R}^{1}=\mathrm{CH}_{2} \mathrm{Ph}$.

30; $\mathrm{R}=\mathrm{R}^{1}=\mathrm{CH}_{2} \mathrm{CH}_{3}$.

31; $\mathrm{R}-\mathrm{R}^{1}=\left(\mathrm{CH}_{2}\right)_{5}$.

Scheme 3 Synthesis of methyl ester of $C_{2}$ - symmetrical and morpholino 1,3,5-triazine tripod derivatives 23-31

Table 1 Minimal inhibitory concentration (MIC) of test compounds in $\mu \mathrm{g} / \mathrm{mL}$

\begin{tabular}{|c|c|c|c|c|c|c|c|}
\hline Test compound & E. coli & S. aureus & C. albicans & Test compound & E. coli & S. aureus & C. albicans \\
\hline Ampicillin & 25 & 12.5 & - & 20 & 100 & $>200$ & $>200$ \\
\hline Clotrimazole & - & - & 12.5 & 21 & 100 & 50 & $>200$ \\
\hline 9 & 50 & 100 & $>200$ & 22 & 100 & 100 & $>200$ \\
\hline 10 & 50 & 12.5 & $>200$ & 23 & 100 & 100 & $>200$ \\
\hline 11 & 50 & 100 & $>200$ & 24 & 100 & 100 & $>200$ \\
\hline 12 & 50 & 50 & $>200$ & 25 & 100 & 12.5 & $>200$ \\
\hline 13 & 25 & 25 & $>200$ & 26 & 100 & 100 & $>200$ \\
\hline 14 & 25 & 100 & $>200$ & 27 & 100 & 100 & $>200$ \\
\hline 15 & 50 & $>200$ & $>200$ & 28 & 100 & $>200$ & $>200$ \\
\hline 16 & 100 & 12.5 & $>200$ & 29 & 100 & $>200$ & $>200$ \\
\hline 17 & 100 & 100 & $>200$ & 30 & 100 & 12.5 & $>200$ \\
\hline 18 & 100 & $>200$ & $>200$ & 31 & 100 & 100 & $>200$ \\
\hline 19 & $>200$ & 100 & $>200$ & & & & \\
\hline
\end{tabular}

broad singlet (br s), doublet (d), doublet of doublets (dd), triplet $(\mathrm{t})$, doublet of triplets $(\mathrm{dt})$, quartet $(\mathrm{q})$, sextet $(\mathrm{sex})$ and multiplet $(\mathrm{m})$. Elemental analyses were performed on a Perkin-Elmer 2400 elemental analyzer, and the values found were within $\pm 0.3 \%$ of the theoretical values. Mass spectra (MS) were recorded on a QP 1000EX/MS Shimatzu Corp by using electron impact (EI) at $70 \mathrm{eV}$. The antimicrobial activity and invitro cytotoxicity were carried at the lab of Prof. Adnan Bekhit, Faculty of Pharmacy, Alexandria University.

\section{General procedure for the synthesis}

of 2-substituted-4,6-dichloro-1,3,5-triazine derivatives (3-8)

To a solution of cyanuric chloride $(1.01 \mathrm{~g}, 5.5 \mathrm{mmol})$ in methylene chloride $(10 \mathrm{~mL})$, amine $(5 \mathrm{mmol})$ and sodium carbonate $(1.06 \mathrm{~g}, 10 \mathrm{mmol})$ were added. The mixture 
Table 2 Minimum inhibitory concentrations (MIC $\mu \mathrm{g} / \mathrm{mL}$ ) for clinical isolates of compounds (10), (13), (14), (16), (25) and (30)

\begin{tabular}{lll}
\hline Test compound & MRSA & E. coli \\
\hline Ampicillin & $>200$ & $>200$ \\
$\mathbf{1 0}$ & $\mathbf{2 5}$ & 100 \\
$\mathbf{1 3}$ & 100 & $\mathbf{2 5}$ \\
$\mathbf{1 4}$ & $>200$ & 100 \\
$\mathbf{1 6}$ & 100 & $>200$ \\
$\mathbf{2 5}$ & 100 & 100 \\
$\mathbf{3 0}$ & $>200$ & $>200$ \\
\hline
\end{tabular}

MRSA methicillin-resistant Staphylococcus aureus

Table $3 \mathrm{CC}_{50}$ values and selectivity index (SI) of the most active compounds on normal VERO

\begin{tabular}{lll}
\hline Test compound & $\left(\mathrm{CC}_{\mathbf{5 0}}\right)^{\mathbf{a}}(\boldsymbol{\mu g} / \mathbf{m L})$ & Selectivity index $(\mathrm{SI})^{\mathbf{b}}$ \\
\hline $\mathbf{1 0}$ & 250 & 10 \\
$\mathbf{1 3}$ & 125 & 5 \\
\hline
\end{tabular}

${ }^{a} \mathrm{CC}_{50}$ is the concentration of compound required to kill $50 \%$ of the fibroblast cells

b The selectivity index (SI) was calculated using the formula, $\mathrm{SI}=\mathrm{CC}_{50} / \mathrm{MIC}$

was vigorously stirred at $0-5{ }^{\circ} \mathrm{C}$ for $3 \mathrm{~h}$. The precipitate was filtered and washed with methylene chloride. The precipitate was dissolved in small amount of water. The solution was neutralized with $1 \mathrm{~N} \mathrm{HCl}$, and the formed precipitate was filtered (Additional file 1).

$N$-(4,6-dichloro-1,3,5-triazin-2-yl)aminobenzoic acid (3) The product was obtained as white solid, $1.36 \mathrm{~g}(95.4 \%)$ yield; $\mathrm{mp}>360{ }^{\circ} \mathrm{C}$ [Lit $\mathrm{mp}>350{ }^{\circ} \mathrm{C}$ ] [47]. ${ }^{1} \mathrm{H}-\mathrm{NMR}$ $\left(500 \mathrm{MHz}, \mathrm{DMSO}-\mathrm{d}_{6}\right): \delta 7.70(\mathrm{~d}, 2 \mathrm{H}, J=8.4 \mathrm{~Hz}, \mathrm{Ar}-\mathrm{H})$, $7.88(\mathrm{~d}, 2 \mathrm{H}, J=8.4 \mathrm{~Hz}, \mathrm{Ar}-\mathrm{H}), 10.88\left(\mathrm{~s}, 1 \mathrm{H}, \mathrm{NH}, \mathrm{D}_{2} \mathrm{O}\right.$ exchangeable).

4,6-dichloro-N-phenyl-1,3,5-triazin-2-amine(4)[48] The product was obtained as white solid, $1.11 \mathrm{~g}$ (92.1\%) yield; mp: $235-238^{\circ} \mathrm{C}$.

N-benzyl-4,6-dichloro-1,3,5-triazin-2-amine(5)[49] The product was obtained as white solid, $1.16 \mathrm{~g}$ (90.8\%) yield; mp: 232-234 (dec.) ${ }^{\circ} \mathrm{C}$; IR (KBr): 3650-2700 (br, OH, acid), 3268 (NH, amine), 1686 (CO, acid) $\mathrm{cm}^{-1}$; ${ }^{1} \mathrm{H}-\mathrm{NMR}$ $\left(500 \mathrm{MHz}, \mathrm{DMSO}-\mathrm{d}_{6}\right): \delta 4.49-4.53\left(\mathrm{~m}, 2 \mathrm{H}, \mathrm{CH}_{2}\right), 7.28-$ $7.31(\mathrm{~m}, 5 \mathrm{H}, \mathrm{Ar}-\mathrm{H}), 9.60\left(\mathrm{t}, 1 \mathrm{H}, J=6.1 \mathrm{~Hz}, \mathrm{NH}, \mathrm{D}_{2} \mathrm{O}\right.$ exchangeable), 11.15 (s, $1 \mathrm{H}, \mathrm{OH}, \mathrm{D}_{2} \mathrm{O}$ exchangeable).

4,6-dichlro-N,N-diethyl-1,3,5-triazin-2-amine(6)[50] The product was obtained as white solid, $1.05 \mathrm{~g}$ (94.6\%) yield; mp: $241-244{ }^{\circ} \mathrm{C} ;{ }^{1} \mathrm{H}-\mathrm{NMR}\left(500 \mathrm{MHz}, \mathrm{DMSO}-\mathrm{d}_{6}\right): \delta 1.09-$ $1.11\left(\mathrm{~m}, 6 \mathrm{H}, 2 \mathrm{CH}_{3}\right), 3.51-3.55\left(\mathrm{~m}, 4 \mathrm{H}, 2 \mathrm{CH}_{2}\right), 11.15(\mathrm{~s}, 1 \mathrm{H}$, $\mathrm{OH}, \mathrm{D}_{2} \mathrm{O}$ exchangeable).

4-(4,6-dichloro-1,3,5-triazin-2-yl)morpholine (7) The product was obtained as white solid, $1.03 \mathrm{~g}(87.3 \%)$ yield; mp: $157-158{ }^{\circ} \mathrm{C}$ [Lit mp 154-156 ${ }^{\circ} \mathrm{C}$ ] [16];. ${ }^{1} \mathrm{H}-\mathrm{NMR}$ $\left(500 \mathrm{MHz}, \mathrm{CDCl}_{3}\right): \delta 3.69\left(\mathrm{t}, 4 \mathrm{H}, J=5.4 \mathrm{~Hz}, 2 \times \mathrm{CH}_{2} \mathrm{~N}\right), \delta$ $3.73\left(\mathrm{t}, 4 \mathrm{H}, J=5.4 \mathrm{~Hz}, 2 \times \mathrm{CH}_{2} \mathrm{O}\right)$.

2,4-dichloro-6-(piperidin-1-yl)-1,3,5-triazine (8) The product was obtained as white solid, $1.04 \mathrm{~g}$ (89.2\%) yield; mp: 143-145 ${ }^{\circ} \mathrm{C}$ [Lit mp $176-178^{\circ} \mathrm{C}$ ] [16]; ${ }^{1} \mathrm{H}-\mathrm{NMR}(500 \mathrm{MHz}$, $\left.\mathrm{CDCl}_{3}\right): \delta 1.60-1.65\left(\mathrm{~m}, 4 \mathrm{H}, 2 \mathrm{CH}_{2}\right), 1.69-1.72\left(\mathrm{~m}, 2 \mathrm{H}, \mathrm{CH}_{2}\right)$, $3.80\left(\mathrm{t}, 4 \mathrm{H}, J=6.1 \mathrm{~Hz}, 2 \mathrm{CH}_{2}-\mathrm{N}\right)$.

\section{General procedure for the synthesis} of 4-((4-chloro-6-substituted-1,3,5-triazin-2-yl)amino) benzoic acid derivatives (9-13)

To a solution of $N$-(4,6-dichloro-1,3,5-triazin-2-yl)aminobenzoic acid 3 (2.0 g, $7.0 \mathrm{mmol})$ and sodium carbonate $(1.78 \mathrm{~g}, 16.8 \mathrm{mmol})$ in distilled water $(20 \mathrm{~mL})$, a solution of amine $(8.4 \mathrm{mmol})$ in dioxane $(5 \mathrm{~mL})$ was added while stirring. The reaction mixture was stirred overnight at room temperature. The reaction mixture was neutralized with $1 \mathrm{~N} \mathrm{HCl}$. The formed precipitate was filtered and washed with water.

4-((4-chloro-6-(phenylamino)-1,3,5-triazin-2-yl)amino) benzoic acid (9) The product was obtained as white solid, 2.38 g (99.5\%) yield; mp: 292-295 (dec.) ${ }^{\circ} \mathrm{C}$; IR (KBr): 3700-2500 (br, OH, acid), 3278 (NH, amine), 1691 (CO, acid) $\mathrm{cm}^{-1} ;{ }^{1} \mathrm{H}-\mathrm{NMR}(500 \mathrm{MHz}$, DMSO-d 6 ): $\delta$ 6.98-7.35 (m, 4H, Ar-H), 7.62-7.97 (m, 5H, Ar-H), 9.38-9.73 (m, 1H, NH, $\mathrm{D}_{2} \mathrm{O}$ exchangeable), 10.25-10.56 (m, $1 \mathrm{H}, \mathrm{NH}, \mathrm{D}_{2} \mathrm{O}$ exchangeable), $12.72\left(\mathrm{~s}, 1 \mathrm{H}, \mathrm{OH}, \mathrm{D}_{2} \mathrm{O}\right.$ exchangeable); ${ }^{13} \mathrm{C}-\mathrm{NMR}$ (125 MHz, DMSO-d 6 ): 120.32, $121.14,128.94,129.15,129.36,130.60,140.23,144.96$, 164.42, 164.52, 167.47, 167.68. Elemental analysis calcd. for $\mathrm{C}_{16} \mathrm{H}_{12} \mathrm{ClN}_{5} \mathrm{O}_{2}$ : C, 56.23; $\mathrm{H}, 3.54 ; \mathrm{Cl}, 10.37 ; \mathrm{N}, 20.49$. Found: C, 56.20; H, 3.59; Cl, 10.33; N, 20.52.

4-((4-(benzylamino)-6-chloro-1,3,5-triazin-2-yl)amino) benzoic acid (10) The product was obtained as white solid, 2.48 g (99.6\%) yield; mp: $272-275$ (dec.) ${ }^{\circ} \mathrm{C}$; IR (KBr): $\mathrm{cm}^{-1} ;{ }^{1} \mathrm{H}-\mathrm{NMR}(500 \mathrm{MHz}$, DMSO-d 6 ): $\delta 4.48-$ $4.52\left(\mathrm{~m}, 2 \mathrm{H}, \mathrm{CH}_{2}\right), 7.20-7.31(\mathrm{~m}, 4 \mathrm{H}, \mathrm{Ar}-\mathrm{H}), 7.76-7.84$ $(\mathrm{m}, 5 \mathrm{H}, \mathrm{Ar}-\mathrm{H}), 8.70-8.83\left(\mathrm{~m}, 1 \mathrm{H}, \mathrm{NH}, \mathrm{D}_{2} \mathrm{O}\right.$ exchangeable), $10.31-10.35$ (m, 1H, NH, $\mathrm{D}_{2} \mathrm{O}$ exchangeable), 12.61 (s, $1 \mathrm{H}, \mathrm{OH}, \mathrm{D}_{2} \mathrm{O}$ exchangeable); ${ }^{13} \mathrm{C}-\mathrm{NMR}(125 \mathrm{MHz}$, DMSO-d ${ }_{6}$ ): 44.2, 119.69, 125.01, 127.31, 127.87, 128.94, 130.62 , 166.00, 166.18, 167.51, 168.60. Elemental analysis calcd. for $\mathrm{C}_{17} \mathrm{H}_{14} \mathrm{ClN}_{5} \mathrm{O}_{2}: \mathrm{C}, 57.39 ; \mathrm{H}, 3.97 ; \mathrm{Cl}, 9.96 ; \mathrm{N}$, 19.68. Found: C, 57.29; H, 4.03; Cl, 9.98; N, 19.64 . 
4-((4-chloro-6-(diethylamino)-1,3,5-triazin-2-yl)amino) benzoic acid (11) The product was obtained as white solid, $2.04 \mathrm{~g}$ (90.6\%) yield; mp: $220-222$ (dec.) ${ }^{\circ} \mathrm{C}$; IR (KBr): 3700-2500 (br, OH, acid), 3285 (NH, amine), 1689 (CO, acid) $\mathrm{cm}^{-1} ;{ }^{1} \mathrm{H}-\mathrm{NMR}(500 \mathrm{MHz}$, DMSO-d 6 ): $\delta 1.09$ $\left(\mathrm{t}, 3 \mathrm{H}, J=6.9 \mathrm{~Hz}, \mathrm{CH}_{3}\right), 1.15\left(\mathrm{t}, 3 \mathrm{H}, J=6.9 \mathrm{~Hz}, \mathrm{CH}_{3}\right)$, 3.50-3.53 (m, 4H, 2CH $\mathrm{CH}_{2}, 7.79(\mathrm{~d}, 2 \mathrm{H}, J=8.4 \mathrm{~Hz}, \mathrm{Ar}-\mathrm{H})$, $7.86(\mathrm{~d}, 2 \mathrm{H}, J=8.4 \mathrm{~Hz}, \mathrm{Ar}-\mathrm{H}), 10.34\left(\mathrm{~s}, 1 \mathrm{H}, \mathrm{NH}, \mathrm{D}_{2} \mathrm{O}\right.$ exchangeable), 12.69 (s, $1 \mathrm{H}, \mathrm{OH}, \mathrm{D}_{2} \mathrm{O}$ exchangeable); ${ }^{13} \mathrm{C}-$ NMR (125 MHz, DMSO-d ${ }_{6}$ ): 13.01, 13.47, 42.01, 42.47, 119.50, 125.03, 130.73, 143.78, 163.87, 164.12, 167.51, 168.83. EIMS $(\mathrm{m} / \mathrm{z}): 321.139\left(\mathrm{M}^{+}\right)$; elemental analysis calcd. for $\mathrm{C}_{14} \mathrm{H}_{16} \mathrm{ClN}_{5} \mathrm{O}_{2}$ : C, 52.26; $\mathrm{H}, 5.01 ; \mathrm{Cl}, 11.02 ; \mathrm{N}$, 21.77. Found: C, 52.30; H, 5.05; Cl, 10.98; N, 21.80.

4-((4-chloro-6-morpholino-1,3,5-triazin-2-yl)amino)benzoic acid (12) The product was obtained as white solid, 2.14 g (91.1\%) yield; mp: $308-311$ (dec.) ${ }^{\circ} \mathrm{C}$; IR (KBr): 3700-2500 (br, OH, acid), 3413(NH, amine), 1684 (CO, acid) $\mathrm{cm}^{-1}$; ${ }^{1} \mathrm{H}-\mathrm{NMR}\left(500 \mathrm{MHz}, \mathrm{DMSO}-\mathrm{d}_{6}\right): \delta 3.58-3.62$ $\left(\mathrm{m}, 4 \mathrm{H}, 2 \mathrm{CH}_{2}\right), 3.65-3.69\left(\mathrm{~m}, 4 \mathrm{H}, 2 \mathrm{CH}_{2}\right), 7.77-7.86(\mathrm{~m}$, $4 \mathrm{H}, \mathrm{Ar}-\mathrm{H}), 10.41$ (s, $1 \mathrm{H}, \mathrm{NH}, \mathrm{D}_{2} \mathrm{O}$ exchangeable), 12.61 (s, $1 \mathrm{H}, \mathrm{OH}, \mathrm{D}_{2} \mathrm{O}$ exchangeable); ${ }^{13} \mathrm{C}-\mathrm{NMR}(125 \mathrm{MHz}$, DMSO- $\mathrm{d}_{6}$ ): 43.91, 66.54, 119.02, 125.26, 130.81, 143.46, 164.50, 165.17, 167.49, 167.66. Elemental analysis calcd. for $\mathrm{C}_{14} \mathrm{H}_{14} \mathrm{ClN}_{5} \mathrm{O}_{3}:$ C, 50.08; H, 4.20; Cl, 10.56; N, 20.86. Found: C, 50.17; H, 4.15; Cl, 10.51; N, 20.98 .

4-((4-chloro-6-(piperidin-1-yl)-1,3,5-triazin-2-yl)amino) benzoic acid (13) The product was obtained as white solid, $2.11 \mathrm{~g}(90.3 \%)$ yield; mp: $284-287$ (dec.) ${ }^{\circ} \mathrm{C}$; IR (KBr): 3700-2600 (br, OH, acid), 3269 (NH, amine), 1689 (CO, acid) $\mathrm{cm}^{-1}$; ${ }^{1} \mathrm{H}-\mathrm{NMR}\left(500 \mathrm{MHz}, \mathrm{DMSO}-\mathrm{d}_{6}\right): \delta 0.85-0.88$ (m, 3H, pip), 1.51-1.54 (m, 2H, $\mathrm{CH}_{2}$-pip), 3.18-3.24 (m, $3 \mathrm{H}$, pip), 7.80-7.84 (m, 4H, Ar-H), 10.26-10.37 (m, $1 \mathrm{H}$, $\mathrm{NH}, \mathrm{D}_{2} \mathrm{O}$ exchangeable), $12.72\left(\mathrm{~s}, 1 \mathrm{H}, \mathrm{OH}, \mathrm{D}_{2} \mathrm{O}\right.$ exchangeable); ${ }^{13} \mathrm{C}-\mathrm{NMR}(125 \mathrm{MHz}$, DMSO-d 6 ): 11.92, 22.36, 42.96, $119.63,125.05,130.68,143.80,164.27,165.91,167.51$, 168.43. Elemental analysis calcd. for $\mathrm{C}_{15} \mathrm{H}_{16} \mathrm{ClN}_{5} \mathrm{O}_{2}: \mathrm{C}$, 53.98; H, 4.83; Cl, 10.62; N, 20.98. Found: C, 53.91; H, 4.89; $\mathrm{Cl}, 10.56 ; \mathrm{N}, 21.03$.

\section{General procedure for the synthesis \\ of 4-((4,6-disubstituted-1,3,5-triazin-2-yl)amino)benzoic acid derivatives (14-18)}

Method A Conventional procedure: To a solution of $N$-(4,6-dichloro-1,3,5-triazin-2-yl)aminobenzoic acid 3 (0.71 g, $2.5 \mathrm{mmol})$ and sodium carbonate $(0.95 \mathrm{~g}$, $9.0 \mathrm{mmol})$ in distilled water $(20 \mathrm{~mL})$, a solution of amine $(6.25 \mathrm{mmol})$ in dioxane $(5 \mathrm{~mL})$ was added while stirring. The reaction mixture was stirred at room temperature for $2 \mathrm{~h}$ then refluxed at $70-80{ }^{\circ} \mathrm{C}$ for $8-10 \mathrm{~h}$. The reaction mixture was neutralized with $1 \mathrm{~N} \mathrm{HCl}$ after cooling. The corresponding crude products were filtered, dried, and recrystallized from ethanol.

Method $B$ Microwave-irradiation: Employing a multimode reactor (Synthos 3000, Aton Paar GmbH, 1400 W maximum magnetron), the initial step was conducted with 4-Teflon vessels rotor (MF 100) that allow processing four reactions under the same conditions. Each vessel has $N$-(4,6-dichloro-1,3,5-triazin-2-yl)aminobenzoic acid $3(0.71 \mathrm{~g}, 2.5 \mathrm{mmol})$ and sodium carbonate $(0.95 \mathrm{~g}$, $9.0 \mathrm{mmol})$ mixed with the appropriate amine $(6.25 \mathrm{mmol})$ in $3 \mathrm{~mL}$ dioxane/water (1:1). The individual vessels were purged with nitrogen gas for $5 \mathrm{~min}$ and then were placed in the corresponding rotor, fixed by screwing down the upper rotor place, and finally the rotor was closed with a protective hood. The vessels were heated for $5 \mathrm{~min}$ at $100{ }^{\circ} \mathrm{C}$ and held at the same temperature for a further 5 min ( 2 bar pressure, $400 \mathrm{~W})$. Cooling was accomplished by a fan $(5 \mathrm{~min})$. The reaction mixture was neutralized with $1 \mathrm{~N} \mathrm{HCl}$ after cooling. The corresponding crude products were filtered, dried, and recrystallized from ethanol (Additional file 1).

4-((4,6-bis(phenylamino)-1,3,5-triazin-2-yl)amino)benzoic acid (14) The product was obtained as white solid, Method A 0.75 g (75.3\%) yield; Method B 0.91 g (91\%) yield; mp: $318-320{ }^{\circ} \mathrm{C}$; IR (KBr): $3600-2700$ (br, OH, acid), 3413 (NH, amine), 1686 (CO, acid) $\mathrm{cm}^{-1}$; ${ }^{1} \mathrm{H}-\mathrm{NMR}$ $\left(500 \mathrm{MHz}, \mathrm{DMSO}-\mathrm{d}_{6}\right): \delta 7.27-7.83(\mathrm{~m}, 14 \mathrm{H}, \mathrm{Ar}-\mathrm{H})$, 9.34-9.89 (m, 3H, NH, $\mathrm{D}_{2} \mathrm{O}$ exchangeable), $12.53(\mathrm{~s}, 1 \mathrm{H}$, $\mathrm{OH}, \mathrm{D}_{2} \mathrm{O}$ exchangeable); ${ }^{13} \mathrm{C}-\mathrm{NMR}(125 \mathrm{MHz}$, DMSO$\left.\mathrm{d}_{6}\right): 119.48,121.14,122.83,124.07,128.94,130.54,140.27$, 144.96, 164.52, 164.63, 167.76. Elemental analysis calcd. for $\mathrm{C}_{22} \mathrm{H}_{18} \mathrm{~N}_{6} \mathrm{O}_{2}$ : C, 66.32; H, 4.55; N, 21.09. Found: C, 66.12; H, 4.65; N, 21.01 .

4-((4,6-bis(benzylamino)-1,3,5-triazin-2-yl)amino)benzoic acid (15) The product was obtained as white solid, Method A $0.85 \mathrm{~g}(79.2 \%)$ yield; Method B $0.95 \mathrm{~g}$ (88.5\%) yield; mp: $308-310{ }^{\circ} \mathrm{C}$; IR (KBr): $3600-2800$ (br, OH, acid), 3441 (NH, amine), 1677 (CO, acid) $\mathrm{cm}^{-1}$; ${ }^{1} \mathrm{H}-\mathrm{NMR}$ $\left(500 \mathrm{MHz}, \mathrm{DMSO}-\mathrm{d}_{6}\right): \delta 4.42-4.49\left(\mathrm{~m}, 4 \mathrm{H}, 2 \mathrm{CH}_{2}\right), 7.18$ $7.77(\mathrm{~m}, 16 \mathrm{H}, 14 \mathrm{Ar}-\mathrm{H}, 2 \mathrm{NH}), 9.25-9.31(\mathrm{~m}, 1 \mathrm{H}, \mathrm{NH}$, $\mathrm{D}_{2} \mathrm{O}$ exchangeable), 11.97 (s, $1 \mathrm{H}, \mathrm{OH}, \mathrm{D}_{2} \mathrm{O}$ exchangeable); ${ }^{13} \mathrm{C}-\mathrm{NMR}\left(125 \mathrm{MHz}, \mathrm{DMSO}-\mathrm{d}_{6}\right.$ ): 43.93, 118.77, 127.03, $127.41,127.62,127.95,128.71,130.43 .141 .07,164.61$, 166.18, 166.27, 167.95. EIMS (m/z): $426.055\left(\mathrm{M}^{+}\right)$; elemental analysis calcd. for $\mathrm{C}_{24} \mathrm{H}_{22} \mathrm{~N}_{6} \mathrm{O}_{2}$ : C, 67.59; $\mathrm{H}, 5.20$; N, 19.71. Found: C, 67.67; H, 5.12; N, 19.81.

4-((4,6-bis(diethylamino)-1,3,5-triazin-2-yl)amino)benzoic acid (16) The product was obtained as white solid, Method A $0.77 \mathrm{~g}(85.9 \%)$ yield; Method B $0.82 \mathrm{~g}(92 \%)$ yield; 
mp: $280-283^{\circ} \mathrm{C}$; IR (KBr): $3700-2700$ (br, OH, acid), 3420 (NH, amine), 1683 (CO, acid) $\mathrm{cm}^{-1} ;{ }^{1} \mathrm{H}-\mathrm{NMR}(500 \mathrm{MHz}$, DMSO- $\mathrm{d}_{6}$ ): $\delta 1.09$ (des. t, $\left.12 \mathrm{H}, 4 \mathrm{CH}_{3}\right), 3.50-3.61(\mathrm{~m}, 8 \mathrm{H}$, $\left.4 \mathrm{CH}_{2}\right), 7.77-7.86(\mathrm{~m}, 4 \mathrm{H}, \mathrm{Ar}-\mathrm{H}), 9.27\left(\mathrm{~s}, 1 \mathrm{H}, \mathrm{NH}, \mathrm{D}_{2} \mathrm{O}\right.$ exchangeable), 12.48 (s, $1 \mathrm{H}, \mathrm{OH}, \mathrm{D}_{2} \mathrm{O}$ exchangeable); ${ }^{13} \mathrm{C}-$ NMR (125 MHz, DMSO- $\mathrm{d}_{6}$ ): 13.77, 13.95, 41.22, 118.52, 119.13, 123.54, 145.84, 164.48, 167.67. EIMS $(\mathrm{m} / \mathrm{z})$ : 358.144 $\left(\mathrm{M}^{+}\right)$; elemental analysis calcd. for $\mathrm{C}_{18} \mathrm{H}_{26} \mathrm{~N}_{6} \mathrm{O}_{2}$ : C, 60.32; H, 7.31; N, 23.45. Found: C, 60.22; H, 7.40; N, 23.52 .

4-((4,6-dimorpholino-1,3,5-triazin-2-yl)amino)benzoic acid (17) The product was obtained as white solid, Method A 0.72 g (74.5\%) yield; Method B 0.85 g (88\%) yield; mp: $292-294{ }^{\circ} \mathrm{C}$; IR (KBr): $3700-2500$ (br, OH, acid), 3438 (NH, amine), 1711 (CO, acid) $\mathrm{cm}^{-1}$; ${ }^{1} \mathrm{H}-\mathrm{NMR}$ (500 MHz, DMSO- $\left.\mathrm{d}_{6}\right): \delta 3.60-3.69\left(\mathrm{~m}, 16 \mathrm{H}, 8 \mathrm{CH}_{2}\right), 7.73$ $(\mathrm{d}, 2 \mathrm{H}, J=8.4 \mathrm{~Hz}, \mathrm{Ar}-\mathrm{H}), 7.83(\mathrm{~d}, 2 \mathrm{H}, J=8.4 \mathrm{~Hz}, \mathrm{Ar}-\mathrm{H})$, 9.74-9.87 (m, $1 \mathrm{H}, \mathrm{NH}, \mathrm{D}_{2} \mathrm{O}$ exchangeable). Elemental analysis calcd. for $\mathrm{C}_{18} \mathrm{H}_{22} \mathrm{~N}_{6} \mathrm{O}_{4}: \mathrm{C}, 55.95 ; \mathrm{H}, 5.74 ; \mathrm{N}, 21.75$. Found: C, 56.01; H, 5.63; N, 21.64.

3.1.3.5.4-((4,6-di(piperidin-1-yl)-1,3,5-triazin-2-yl)amino) benzoic acid (18) The product was obtained as white solid, Method A $0.82 \mathrm{~g}(85.8 \%)$ yield; Method B $0.89 \mathrm{~g}$ (93.1\%) yield; mp: $284-286{ }^{\circ} \mathrm{C}$; IR (KBr): $3700-2500$ (br, $\mathrm{OH}$, acid), $3291\left(\mathrm{NH}\right.$, amine), $1691(\mathrm{CO}$, acid $) \mathrm{cm}^{-1}$; ${ }^{1} \mathrm{H}-\mathrm{NMR}\left(500 \mathrm{MHz}, \mathrm{DMSO}-\mathrm{d}_{6}\right): \delta \quad 0.84-0.87(\mathrm{~m}, 6 \mathrm{H}$, $3 \mathrm{CH}_{2}$-pip), $1.49-1.51$ (m, 4H, 2 $\mathrm{CH}_{2}$-pip), 3.18-3.20 (m, $10 \mathrm{H}, 5 \mathrm{CH}_{2}$-pip), 7.77-7.90 (m, 4H, Ar-H), 9.15-9.55 (m, $1 \mathrm{H}, \mathrm{NH}, \mathrm{D}_{2} \mathrm{O}$ exchangeable), 12.52 (brs, $1 \mathrm{H}, \mathrm{OH}, \mathrm{D}_{2} \mathrm{O}$ exchangeable); ${ }^{13} \mathrm{C}-\mathrm{NMR}$ (125 MHz, DMSO- $\mathrm{d}_{6}$ ): 11.94 , 23.06, 42.31, 118.75, 123.16, 130.52, 145.73, 164.21, 165.89, 166.2, 167.84. Elemental analysis calcd. for $\mathrm{C}_{20} \mathrm{H}_{26} \mathrm{~N}_{6} \mathrm{O}_{2}$ : C, 62.81; H, 6.85; N, 21.97. Found: C, 62.73; H, 6.94; N, 22.02 .

\section{General procedure for the synthesis of 4-((4-substituted-6-morpholino-1,3,5-triazin-2-yl)amino) benzoic acid derivatives (19-22)}

Method A Conventional procedure: To a solution of $N$-(4-chloro-6-substituted-1,3,5-triazin-2-yl)aminobenzoic acid $(2.5 \mathrm{mmol})$ and sodium carbonate $(0.66 \mathrm{~g}$, $6.25 \mathrm{mmol})$ in distilled water $(15 \mathrm{~mL})$, add a solution of morpholine $(0.33 \mathrm{~mL}, 3.75 \mathrm{mmol})$ in dioxane $(5 \mathrm{~mL})$ with stirring. The reaction mixture was stirred at room temperature for $2 \mathrm{~h}$ then refluxed at $70-80{ }^{\circ} \mathrm{C}$ for $8-9 \mathrm{~h}$. The reaction mixture was neutralized with $1 \mathrm{~N} \mathrm{HCl}$ after cooling. The corresponding crude products were filtered, dried, and recrystallized from ethanol.

Method B Microwave-irradiation: Employing a multimode reactor (Synthos 3000, Aton Paar GmbH, 1400 W maximum magnetron); the initial step was conducted with 4-Teflon vessels rotor (MF 100) that allow processing four reactions under the same conditions. Each vessel has $N$-(4-chloro-6-substituted-1,3,5-triazin-2-yl) aminobenzoic acid $(2.5 \mathrm{mmol})$ and sodium carbonate $(0.66 \mathrm{~g}, 6.25 \mathrm{mmol})$ mixed with morpholine $(0.33 \mathrm{~mL}$, $3.75 \mathrm{mmol}$ ) in $3 \mathrm{~mL}$ dioxane/water (1:1). The individual vessels were purged with nitrogen gas for $5 \mathrm{~min}$ and then were placed in the corresponding rotor, fixed by screwing down the upper rotor place, and finally the rotor was closed with a protective hood. The vessels were heated for $5 \mathrm{~min}$ at $100{ }^{\circ} \mathrm{C}$ and held at the same temperature for a further $5 \mathrm{~min}(\sim 2$ bar pressure, $400 \mathrm{~W})$. Cooling was accomplished by a fan $(5 \mathrm{~min})$. The reaction mixture was neutralized with $1 \mathrm{~N} \mathrm{HCl}$ after cooling. The corresponding crude products were filtered, dried, and recrystallized from ethanol.

4-((4-morpholino-6-(phenylamino)-1,3,5-triazin-2-yl) amino)benzoic acid (19) The product was obtained as white solid, Method A $0.88 \mathrm{~g}(89.7 \%)$ yield; Method B $0.90 \mathrm{~g}$ (92\%) yield; mp: $317-320$ (dec.) ${ }^{\circ} \mathrm{C}$; IR (KBr): 3750 2700 (br, OH, acid), 3410 (NH, amine), 1687 (CO, acid) $\mathrm{cm}^{-1} ;{ }^{1} \mathrm{H}-\mathrm{NMR}\left(500 \mathrm{MHz}, \mathrm{DMSO}-\mathrm{d}_{6}\right): \delta 3.64$ (des. $\mathrm{t}, 4 \mathrm{H}$, $2 \mathrm{CH}_{2}-\mathrm{N}$ ), 3.73 (des. $\left.\mathrm{t}, 4 \mathrm{H}, 2 \mathrm{CH}_{2}-\mathrm{O}\right), 6.95-7.96(\mathrm{~m}, 9 \mathrm{H}$, Ar-H), 9.28-9.34 (m, 1H, NH, $\mathrm{D}_{2} \mathrm{O}$ exchangeable), 9.55$9.59\left(\mathrm{~m}, 1 \mathrm{H}, \mathrm{NH}, \mathrm{D}_{2} \mathrm{O}\right.$ exchangeable), $12.46(\mathrm{~s}, 1 \mathrm{H}, \mathrm{OH}$, $\mathrm{D}_{2} \mathrm{O}$ exchangeable); ${ }^{13} \mathrm{C}-\mathrm{NMR}$ (125 MHz, DMSO- $\mathrm{d}_{6}$ ): 44.01, 66.52, 119.29, 121.02, 122.61,123.94, 128.96, 130.62, 140.40, 145.04, 164.54, 164.61, 165.22, 167.76. Elemental analysis calcd. for $\mathrm{C}_{20} \mathrm{H}_{20} \mathrm{~N}_{6} \mathrm{O}_{3}: \mathrm{C}, 61.21 ; \mathrm{H}, 5.14 ; \mathrm{N}, 21.42$. Found: C, 61.31; H, 5.04; N, 21.32.

4-((4-(benzylamino)-6-morpholino-1,3,5-triazin-2-yl) amino)benzoic acid (20) The product was obtained as white solid, Method A $0.85 \mathrm{~g}$ (83.7\%) yield; Method B $0.92 \mathrm{~g}(90.6 \%)$ yield; mp: $229-232{ }^{\circ} \mathrm{C}$; IR (KBr): 3700-2600 (br, OH, acid), 3410 (NH, amine), 1687 (CO, acid) cm ${ }^{-1}$; ${ }^{1} \mathrm{H}-\mathrm{NMR}\left(500 \mathrm{MHz}\right.$, DMSO-d $\left.\mathrm{d}_{6}\right): \delta 3.57-3.74(\mathrm{~m}, 8 \mathrm{H}$, $\left.4 \mathrm{CH}_{2}\right), 4.45-4.50\left(\mathrm{~m}, 2 \mathrm{H}\right.$, benzyl $\left.\mathrm{CH}_{2}\right), 7.18-7.71(\mathrm{~m}, 4 \mathrm{H}$, $\mathrm{Ar}-\mathrm{H}), 7.69-7.85$ (m, 5H, Ar-H), 9.49-9.73 (m, 1H, NH, $\mathrm{D}_{2} \mathrm{O}$ exchangeable), 12.40 (s, $1 \mathrm{H}, \mathrm{OH}, \mathrm{D}_{2} \mathrm{O}$ exchangeable); ${ }^{13} \mathrm{C}-\mathrm{NMR}$ (125 MHz, DMSO-d 6 ): 43.93, 44.09, 66.48, $119.00,123.60,127.18,128.73,130.56,140.75,145.12$, 164.54, 164.61, 165.22, 167.66. Elemental analysis calcd. for $\mathrm{C}_{21} \mathrm{H}_{22} \mathrm{~N}_{6} \mathrm{O}_{3}$ : C, 62.06; $\mathrm{H}, 5.46 ; \mathrm{N}, 20.68$. Found: C, 62.17; H, 5.58; N, 20.48.

4-((4-(diethylamino)-6-morpholino-1,3,5-triazin-2-yl) amino)benzoic acid (21) The product was obtained as white solid, Method A $0.78 \mathrm{~g}(83.8 \%)$ yield; Method B $0.84 \mathrm{~g}(90.2 \%)$ yield; mp: $258-261{ }^{\circ} \mathrm{C}$; IR (KBr): $3700-2500$ (br, OH, acid), 3391 (NH, amine), $1687\left(\mathrm{CO}\right.$, acid) $\mathrm{cm}^{-1}$; 
${ }^{1} \mathrm{H}-\mathrm{NMR}\left(500 \mathrm{MHz}\right.$, DMSO-d ${ }_{6}$ ): $\delta 1.10$ (des. $\mathrm{t}, 6 \mathrm{H}, 2$ $\left.\mathrm{CH}_{3}\right), 3.49-3.52\left(\mathrm{~m}, 4 \mathrm{H}, 2 \mathrm{CH}_{2}\right), 3.58-3.73\left(\mathrm{~m}, 8 \mathrm{H}, 4 \mathrm{CH}_{2}\right)$, $7.81(\mathrm{~m}, 4 \mathrm{H}, \mathrm{Ar}-\mathrm{H}), 9.45\left(\mathrm{~m}, 1 \mathrm{H}, \mathrm{NH}, \mathrm{D}_{2} \mathrm{O}\right.$ exchangeable), 12.52 (s, $1 \mathrm{H}, \mathrm{OH}, \mathrm{D}_{2} \mathrm{O}$ exchangeable); ${ }^{13} \mathrm{C}-\mathrm{NMR}$ (125 MHz, DMSO-d $\mathrm{d}_{6}$ ): 13.60, 13.77, 41.38, 43.90, 66.52, $118.79,123.46,130.66,145.35,164.02,164.69,164.88$, 167.68. Elemental analysis calcd. for $\mathrm{C}_{18} \mathrm{H}_{24} \mathrm{~N}_{6} \mathrm{O}_{3}$ : C, $58.05 ; \mathrm{H}, 6.50 ; \mathrm{N}, 22.57$. Found: C, 58.00; H, 6.48; N, 22.52 .

4-((4-morpholino-6-(piperidin-1-yl)-1,3,5-triazin-2-yl) amino)benzoic acid (22) The product was obtained as white solid, Method A $0.79 \mathrm{~g}(82.2 \%)$ yield; Method B $0.88 \mathrm{~g}$ (91.6\%) yield; $\mathrm{mp:} 248-250{ }^{\circ} \mathrm{C}$; IR (KBr): $3700-$ 2700 (br, OH, acid), 3292 (NH, amine), 1656 (CO, acid) $\mathrm{cm}^{-1} ;{ }^{1} \mathrm{H}-\mathrm{NMR}\left(500 \mathrm{MHz}, \mathrm{DMSO}-\mathrm{d}_{6}\right): \delta 0.85-0.88(\mathrm{~m}$, $3 \mathrm{H}$, pip), $1.50-1.51$ (m, $2 \mathrm{H}, \mathrm{CH}_{2}$-pip), 3.21-3.68 (m, $13 \mathrm{H}$, $4 \mathrm{CH}_{2}$-mor, 5H-pip), 7.81-7.85 (m, 4H, Ar-H), 9.54-9.65 (m, $1 \mathrm{H}, \mathrm{NH}, \mathrm{D}_{2} \mathrm{O}$ exchangeable), 12.41 (brs, $1 \mathrm{H}, \mathrm{OH}, \mathrm{D}_{2} \mathrm{O}$ exchangeable); ${ }^{13} \mathrm{C}-\mathrm{NMR}\left(125 \mathrm{MHz}, \mathrm{DMSO}-\mathrm{d}_{6}\right): 12.02$, 23.01, 42.64, 44.01, 66.48, 119.09, 123.77, 130.62, 145.87, 164.48, 165.91, 167.65. Elemental analysis calcd. for $\mathrm{C}_{19} \mathrm{H}_{24} \mathrm{~N}_{6} \mathrm{O}_{3}: \mathrm{C}, 59.36 ; \mathrm{H}, 6.29 ; \mathrm{N}, 21.86$. Found: C, 59.31; $\mathrm{H}, 6.22 ; \mathrm{N}, 21.75$.

\section{General procedure for the synthesis of methyl 4-((4,6-disubsituted-1,3,5-triazin-2-yl)amino)benzoic acid derivatives (23-31)}

Concentrated sulphuric acid $(0.5 \mathrm{~mL}, 99 \%)$ was added to a cooled suspension of $N$-(4,6-disubsituted-1,3,5-triazin2-yl)aminobenzoic acid $(1 \mathrm{mmol})$ in methanol $(20 \mathrm{~mL})$. The reaction mixture was refluxed for $8-10 \mathrm{~h}$. The reaction mixture was cooled and poured into sodium bicarbonate solution. The corresponding crude products were filtered, dried, and recrystallized from ethanol (Additional file 1).

Methyl 4-((4,6-bis(phenylamino)-1,3,5-triazin-2-yl)amino) benzoate (23) The product was obtained as white solid, 0.39 g (94.6\%) yield; mp: $250-252^{\circ} \mathrm{C}$; IR (KBr): $3400(\mathrm{NH}$, amine), 1705 (CO, ester) $\mathrm{cm}^{-1}$; ${ }^{1} \mathrm{H}-\mathrm{NMR}(500 \mathrm{MHz}$, DMSO-d $\left.\mathrm{d}_{6}\right): \delta 3.80\left(\mathrm{~s}, 3 \mathrm{H}, \mathrm{OCH}_{3}\right), 7.27-7.85(\mathrm{~m}, 14 \mathrm{H}$, Ar-H), 9.37-9.95 (m, 3H, NH, $\mathrm{D}_{2} \mathrm{O}$ exchangeable); ${ }^{13} \mathrm{C}-$ NMR (125 MHz, DMSO-d 6 ): 52.35, 119.57, 121.16, 122.82, $128.96,129.34,130.41,140.19,145.35,164.42,164.52$, 166.56. Elemental analysis calcd. for $\mathrm{C}_{23} \mathrm{H}_{20} \mathrm{~N}_{6} \mathrm{O}_{2}$ : C, 66.98; H, 4.89; N, 20.38. Found: C, 66.83; H, 4.77; N, 20.26.

Methyl 4-((4,6-bis(benzylamino)-1,3,5-triazin-2-yl)amino) benzoate (24) The product was obtained as white solid, $0.44 \mathrm{~g}(99.8 \%)$ yield; mp: $272-274{ }^{\circ} \mathrm{C}$; IR (KBr): $3413(\mathrm{NH}$, amine), 1711 (CO, ester) $\mathrm{cm}^{-1}$; ${ }^{1} \mathrm{H}-\mathrm{NMR}(500 \mathrm{MHz}$, DMSO- $\left.\mathrm{d}_{6}\right): \delta 3.76\left(\mathrm{~s}, 3 \mathrm{H}, \mathrm{OCH}_{3}\right), 4.42-4.48\left(\mathrm{~m}, 4 \mathrm{H}, 2 \mathrm{CH}_{2}\right)$, 7.18-7.69 (m, 16H, 14 Ar-H, 2NH), 9.30-9.36 (m, 1H, $\mathrm{NH}, \mathrm{D}_{2} \mathrm{O}$ exchangeable); ${ }^{13} \mathrm{C}-\mathrm{NMR}(125 \mathrm{MHz}$, DMSO- $\left.d_{6}\right): 43.93,52.25,118.87,127.05,127.34,127.41,127.62$, 128.71, 130.31. 141.01, 166.60. Elemental analysis calcd. for $\mathrm{C}_{25} \mathrm{H}_{24} \mathrm{~N}_{6} \mathrm{O}_{2}$ : C, 68.17; H, 5.49; N, 19.08. Found: C, 68.05; $\mathrm{H}, 5.37 ; \mathrm{N}, 19.01$.

Methyl 4-((4,6-bis(diethylamino)-1,3,5-triazin-2-yl)amino) benzoate (25) The product was obtained as white solid, $0.35 \mathrm{~g}(94.0 \%)$ yield; mp: $190-192{ }^{\circ} \mathrm{C}$; IR $(\mathrm{KBr}): 3338(\mathrm{NH}$, amine), 1718 (CO, ester) $\mathrm{cm}^{-1}$; ${ }^{1} \mathrm{H}-\mathrm{NMR}(500 \mathrm{MHz}$, DMSO- $\left.\mathrm{d}_{6}\right): \delta 1.10\left(\mathrm{~m}, 12 \mathrm{H}, 4 \mathrm{CH}_{3}\right), 3.48-3.54(\mathrm{~m}, 8 \mathrm{H}$, $\left.4 \mathrm{CH}_{2}\right), 3.76\left(\mathrm{~s}, 3 \mathrm{H}, \mathrm{OCH}_{3}\right), 7.79-7.88(\mathrm{~m}, 4 \mathrm{H}, \mathrm{Ar}-\mathrm{H}), 9.33$ $\left(\mathrm{s}, 1 \mathrm{H}, \mathrm{NH}, \mathrm{D}_{2} \mathrm{O}\right.$ exchangeable); ${ }^{13} \mathrm{C}-\mathrm{NMR}(125 \mathrm{MHz}$, DMSO- $\mathrm{d}_{6}$ ): 13.77, $13.95,41.24,52.19,118.60,121.84$, $130.41,146.24,164.46,166.60$. Elemental analysis calcd. for $\mathrm{C}_{19} \mathrm{H}_{28} \mathrm{~N}_{6} \mathrm{O}_{2}$ : C, 61.27; $\mathrm{H}, 7.58 ; \mathrm{N}, 22.56$. Found: $\mathrm{C}, 61.15$; $\mathrm{H}, 7.46 ; \mathrm{N}, 22.45$.

Methyl 4-((4,6-dimorpholino-1,3,5-triazin-2-yl)amino)benzoate (26) The product was obtained as white solid, $0.32 \mathrm{~g}$ (79.9\%) yield; mp: $141-144{ }^{\circ} \mathrm{C}$; IR (KBr): 3411 (NH, amine), 1711 (CO, ester) $\mathrm{cm}^{-1} ;{ }^{1} \mathrm{H}-\mathrm{NMR}\left(500 \mathrm{MHz}\right.$, DMSO-d $\left.\mathrm{d}_{6}\right): \delta$ $3.60-3.68\left(\mathrm{~m}, 16 \mathrm{H}, 8 \mathrm{CH}_{2}\right), 3.77\left(\mathrm{~s}, 3 \mathrm{H}, \mathrm{OCH}_{3}\right), 7.81-7.85(\mathrm{~m}$, $4 \mathrm{H}, \mathrm{Ar}-\mathrm{H}), 9.54\left(\mathrm{~s}, 1 \mathrm{H}, \mathrm{NH}, \mathrm{D}_{2} \mathrm{O}\right.$ exchangeable); ${ }^{13} \mathrm{C}-\mathrm{NMR}$ (125 MHz, DMSO-d d $_{6}: 43.88,52.27,66.52,119.08,122.38$, 130.58, 145.54, 164.50, 165.15, 166.63. Elemental analysis calcd. for $\mathrm{C}_{19} \mathrm{H}_{24} \mathrm{~N}_{6} \mathrm{O}_{4}: \mathrm{C}, 56.99 ; \mathrm{H}, 6.04 ; \mathrm{N}, 20.99$. Found: $\mathrm{C}$, 56.87; H, 5.93; N, 20.88.

Methyl 4-((4,6-di(piperidin-1-yl)-1,3,5-triazin-2-yl)amino) benzoate (27) The product was obtained as white solid, 0.35 g (88.3\%) yield; mp: $240-242{ }^{\circ} \mathrm{C}$; IR $(\mathrm{KBr}): 3335(\mathrm{NH}$, amine), 1711 (CO, ester) cm ${ }^{-1} ;{ }^{1} \mathrm{H}-\mathrm{NMR}(500 \mathrm{MHz}$, DMSO$\left.\mathrm{d}_{6}\right): \delta 0.84-0.87\left(\mathrm{~m}, 8 \mathrm{H}, 4 \mathrm{CH}_{2}\right), 1.48-1.50\left(\mathrm{~m}, 4 \mathrm{H}, 2 \mathrm{CH}_{2}\right)$, 3.18-3.29 (m, 8H, 4CH $\mathrm{CH}_{2}$ ), 3.77 (s, $\left.3 \mathrm{H}, \mathrm{OCH}_{3}\right), 7.77-7.93$ (m, 4H, Ar-H), 9.14-9.37 (m, 1H, NH, $\mathrm{D}_{2} \mathrm{O}$ exchangeable); ${ }^{13} \mathrm{C}-\mathrm{NMR}(125 \mathrm{MHz}$, DMSO-d 6 ): 11.98, 23.06, 42.29, 52.23, $118.75,119.31,130.35,146.20,164.46,166.14,166.65$. Elemental analysis calcd. for $\mathrm{C}_{21} \mathrm{H}_{28} \mathrm{~N}_{6} \mathrm{O}_{2}: \mathrm{C}, 63.62 ; \mathrm{H}, 7.12 ; \mathrm{N}$, 21.20. Found: C, 63.53; H, 7.05; N, 21.09 .

Methyl4-((4-morpholino-6-(phenylamino)-1,3,5-triazin-2-yl) amino)benzoate (28) The product was obtained as white solid, 0.28 g (69.0\%) yield; mp: $232-234{ }^{\circ} \mathrm{C}$; IR (KBr): 3404(NH, amine), 1709 (CO, ester) $\mathrm{cm}^{-1}$; ${ }^{1} \mathrm{H}-\mathrm{NMR}$ (500 MHz, DMSO-d $)_{6}$ : $\delta 3.64$ (des. $\mathrm{t}, 4 \mathrm{H}, 2 \mathrm{CH}_{2}-\mathrm{N}$ ), 3.73 (des. t, $\left.4 \mathrm{H}, 2 \mathrm{CH}_{2}-\mathrm{O}\right), 3.77\left(\mathrm{~s}, 3 \mathrm{H}, \mathrm{OCH}_{3}\right), 6.95-7.96(\mathrm{~m}$, $9 \mathrm{H}, \mathrm{Ar}-\mathrm{H}), 9.29-9.35\left(\mathrm{~m}, 1 \mathrm{H}, \mathrm{NH}, \mathrm{D}_{2} \mathrm{O}\right.$ exchangeable), 9.61-9.71 (m, 1H, NH, $\mathrm{D}_{2} \mathrm{O}$ exchangeable); ${ }^{13} \mathrm{C}-\mathrm{NMR}$ (125 MHz, DMSO-d $\left.\mathrm{d}_{6}\right)$ 44.03, 52.31, 66.52, 119.34, 119.53, $120.77,122.61,128.96,130.48,140.39,145.48,164.52$, 165.20, 166.59. Elemental analysis calcd. for $\mathrm{C}_{21} \mathrm{H}_{22} \mathrm{~N}_{6} \mathrm{O}_{3}$ : C, 62.06; H, 5.46; N, 20.68. Found: C, 61.97; H, 5.33; N, 20.57 . 
Methyl4-((4-(benzylamino)-6-morpholino-1,3,5-triazin-2-yl) amino)benzoate (29) The product was obtained as white solid, $0.34 \mathrm{~g}(80.9 \%)$ yield; mp: $155-158{ }^{\circ} \mathrm{C}$; IR (KBr): 3340 (NH, amine), 1714 (CO, ester) $\mathrm{cm}^{-1}$; ${ }^{1} \mathrm{H}-\mathrm{NMR}(500 \mathrm{MHz}$, DMSO-d $\left.{ }_{6}\right): \delta 3.60-3.66\left(\mathrm{~m}, 8 \mathrm{H}, 4 \mathrm{CH}_{2}\right), 3.77\left(\mathrm{~s}, 3 \mathrm{H}, \mathrm{OCH}_{3}\right)$, 4.45-4.50 (dd, $2 \mathrm{H}, J=23.7,6.1 \mathrm{~Hz}$, benzyl $\left.\mathrm{CH}_{2}\right), 7.17-7.30$ (m, 4H, Ar-H), 7.63-7.88 (m, 5H, Ar-H), 9.42-9.47 (m, $1 \mathrm{H}, \mathrm{NH}, \mathrm{D}_{2} \mathrm{O}$ exchangeable); ${ }^{13} \mathrm{C}-\mathrm{NMR}(125 \mathrm{MHz}$, DMSO$\left.\mathrm{d}_{6}\right)$ : 43.84, 44.07, 52.25, 66.50, 79.63, 118.98, 122.24, 127.15, 127.34, 127.95, 128.71, 130.45, 140.88, 145.84, 164.57, 165.18, $166.48,166.62$. EIMS $(\mathrm{m} / \mathrm{z})$ : $420.036\left(\mathrm{M}^{+}\right)$; elemental analysis calcd. for $\mathrm{C}_{22} \mathrm{H}_{24} \mathrm{~N}_{6} \mathrm{O}_{3}: \mathrm{C}, 62.84 ; \mathrm{H}, 5.75 ; \mathrm{N}, 19.99$. Found: C, 62.76; H, 5.65; N, 19.87 .

Methyl 4-((4-(diethylamino)-6-morpholino-1,3,5-triazin-2-yl) amino)benzoate (30) The product was obtained as white solid, $0.28 \mathrm{~g}$ (72.5\%) yield; mp: $151-153{ }^{\circ} \mathrm{C}$; IR (KBr): 3337 (NH, amine), 1717 (CO, ester) cm ${ }^{-1}$; ${ }^{1} \mathrm{H}-\mathrm{NMR}(500 \mathrm{MHz}$, DMSO-d $\mathrm{d}_{6}$ ): $\delta 1.10$ (des. t, $\left.6 \mathrm{H}, 2 \mathrm{CH}_{3}\right), 3.49-3.54(\mathrm{~m}, 4 \mathrm{H}$, $\left.2 \mathrm{CH}_{2}\right), 3.59-3.68\left(\mathrm{~m}, 8 \mathrm{H}, 4 \mathrm{CH}_{2}\right), 3.77\left(\mathrm{~s}, 3 \mathrm{H}, \mathrm{OCH}_{3}\right), 7.82-$ $7.86(\mathrm{~m}, 4 \mathrm{H}, \mathrm{Ar}-\mathrm{H}), 9.44\left(\mathrm{~s}, 1 \mathrm{H}, \mathrm{NH}, \mathrm{D}_{2} \mathrm{O}\right.$ exchangeable); ${ }^{13} \mathrm{C}-\mathrm{NMR}\left(125 \mathrm{MHz}\right.$, DMSO-d $\left.{ }_{6}\right): 13.62,13.77,41.30,43.86$, 66.54, 79.70, 118.81, 122.11, 130.48, 145.92, 164.50, 165.22, 166.58. Elemental analysis calcd. for $\mathrm{C}_{19} \mathrm{H}_{26} \mathrm{~N}_{6} \mathrm{O}_{3}$ : C, 59.05; $\mathrm{H}, 6.78$; N, 21.75. Found: C, 59.01; H, 6.67; N, 21.65 .

Methyl4-((4-morpholino-6-(piperidin-1-yl)-1,3,5-triazin-2-yl) amino)benzoate (31) The product was obtained as white solid, $0.26 \mathrm{~g}(65.3 \%)$ yield; $\mathrm{mp}: 140-142{ }^{\circ} \mathrm{C}$; IR $(\mathrm{KBr}): 3342$ (NH, amine), 1714 (CO, ester) $\mathrm{cm}^{-1} ;{ }^{1} \mathrm{H}-\mathrm{NMR}(500 \mathrm{MHz}$, DMSO- $\mathrm{d}_{6}$ ): $\delta 1.47-1.59$ (m, 6H, $3 \mathrm{CH}_{2}$, pip), 3.54-3.69 (m, $12 \mathrm{H}, 4 \mathrm{CH}_{2}$ mor, $2 \mathrm{CH}_{2}$ pip), 3.77 (s, $\left.3 \mathrm{H}, \mathrm{OCH}_{3}\right), 7.80-7.82$ $(\mathrm{m}, 4 \mathrm{H}, \mathrm{Ar}-\mathrm{H}), 9.46\left(\mathrm{~s}, 1 \mathrm{H}, \mathrm{NH}, \mathrm{D}_{2} \mathrm{O}\right.$ exchangeable); ${ }^{13} \mathrm{C}-$ NMR (125 MHz, DMSO-d ${ }_{6}$ ): 24.86, 25.94, 43.91, 44.22, 52.23, 66.56, 118.94, 122.21, 130.54, 145.78, 164.57, 164.73, 165.36, 166.58. Elemental analysis calcd. for $\mathrm{C}_{20} \mathrm{H}_{26} \mathrm{~N}_{6} \mathrm{O}_{3}$ : C, 60.29; H, 6.58; N, 21.09. Found: C, 60.20; H, 6.49; N, 21.02 .

\section{Biology}

The microdilution susceptibility test in Müller-Hinton Broth (Oxoid) and Sabouraud Liquid Medium (Oxoid) were used for the determination of antibacterial and antifungal activity [45]. The utilized test organisms were: Escherichia coli (E. coli) ATCC 25922 as an example of Gram-negative bacteria, Staphylococcus aureus (S. aureus) ATCC 19433 as an example of Gram-positive bacteria and Candida albicans (C. albicans) as yeastlike fungi. Ampicillin trihydrate and clotrimazole were used as standard antibacterial and antifungal agents, respectively. Solutions of the test compounds, ampicillin trihydrate and clotrimazole were prepared in DMSO to a concentration of $1600 \mu \mathrm{g} / \mathrm{mL}$. Twofold dilutions of the compounds were prepared $(800,400, \ldots 6.25 \mu \mathrm{g} /$ $\mathrm{mL}$ ). Microorganism suspensions at $106 \mathrm{CFU} / \mathrm{mL}$ (Colony Forming Unit $/ \mathrm{mL}$ ) concentrations were inoculated to the corresponding wells. Plates were incubated at $36{ }^{\circ} \mathrm{C}$ for $24-48 \mathrm{~h}$. The incubation chamber was kept sufficiently humid. At the end of the incubation period, the minimal inhibitory concentrations (MIC) were determined.

Invitro cytotoxicity of the test compounds (10) and (13) were carried out with 5ero cell line using Mosmann method with certain modifications as described in the literature [46]. Briefly the cells were incubated for $72 \mathrm{~h}$ with different dilutions of selected compounds using MTT as reagent for the detection of cytotoxicity. Results were expressed as half maximal cytotoxic concentration $\left(C_{50}\right)$ of the fibroblast cells in $\mu \mathrm{g} / \mathrm{mL} .50 \%$ cytotoxic concentration $\left(\mathrm{CC}_{50}\right)$ values represent the concentration of compound required to kill $50 \%$ of the fibroblast cells. The selectivity index (SI) was calculated using the formula, $\mathrm{SI}=\mathrm{CC}_{50} / \mathrm{MIC}$.

\section{Conclusions}

Synthesis and characterization of mono-, di- and trisubstituted s-triazine derivatives, containing 4-aminobenzoic acid moiety were described. The 1,3,5-triazine tripod series were prepared by conventional method or by using microwave irradiation, employing a multimode reactor (Synthos 3000, Aton Paar GmbH, 1400 W maximum magnetron). Using microwave irradiation gave the desired products in less time, good yield and higher purity. Esterification of the 4-aminobenzoic acid moiety afforded methyl ester analogues. All synthesized compounds were evaluated for their antimicrobial activity. Where, some of which showed either comparable or $50 \%$ activity of that of ampicillin against S. aureus and E. coli. All compounds had lower antifungal activity than clotrimazole (Canesten ${ }^{\circledR}$, Bayer). Compounds (10), (16), (25) and (30) have antimicrobial activity against S. aureus comparable to that of ampicillin, while the activity of compound (13) is about $50 \%$ of that of ampicillin. Compounds (13) and (14) have antimicrobial activity against E. coli comparable to that of ampicillin, while the activity of compounds (9-12) and (15) is about 50\% of that of ampicillin.

Furthermore, minimum inhibitory concentrations values for clinical isolates of compounds (10), (13), (14), (16), (25) and (30) were tested. Compounds (10) and (13) were more active against MRSA and E. coli than ampicillin. Invitro cytotoxicity results revealed that compounds (10) and (13) were nontoxic up to $250 \mu \mathrm{g} / \mathrm{mL}$ (with $\mathrm{SI}=10$ ) and $125 \mu \mathrm{g} / \mathrm{mL}$ (with $\mathrm{SI}=5$ ), respectively. These results prompted us to further pursue in SAR as our future plane. 


\section{Additional file}

Additional file 1. Additional figures.

\section{Authors' contributions}

The main part of the work was carried out by HHK, with the direct supervision of KMA and SNK. Conceptually the work was designed by KMA, AEF and SNK. All authors read and approved the final manuscript.

\section{Acknowledgements}

This project was funded by the Deanship of Scientific Research (DSR), King Abdulaziz University, Jeddah, under Grant No. (298/363/1434). The authors, therefore, acknowledge with thanks DSR technical and financial support.

\section{Competing interests}

The authors declare that they have no competing interests.

\section{Publisher's Note}

Springer Nature remains neutral with regard to jurisdictional claims in published maps and institutional affiliations.

Received: 11 March 2017 Accepted: 2 May 2017

Published online: 10 May 2017

\section{References}

1. Koç ZE (2011) Complexes of iron (III) and chromium (III) salen and salophen Schiff bases with bridging 1, 3, 5-triazine derived multidirectional ligands. J Heterocycl Chem 48(4):769-775

2. Carofiglio T, Varotto A, Tonellato $U$ (2004) One-pot synthesis of cyanuric acid-bridged porphyrin-porphyrin dyads. J Org Chem 69(23):8121-8124

3. Mooibroek TJ, Gamez P (2007) The s-triazine ring, a remarkable unit to generate supramolecular interactions. Inorg Chim Acta 360(1):381-404

4. Porter JR, Archibald SC, Brown JA, Childs K, Critchley D, Head JC et al (2002) Discovery and evaluation of $\mathrm{N}$-(triazin-1, 3, 5-yl) phenylalanine derivatives as VLA-4 integrin antagonists. Biorg Med Chem Lett. 12(12):1591-1594

5. Mylari BL, Withbroe GJ, Beebe DA, Brackett NS, Conn EL, Coutcher JB et al (2003) Design and synthesis of a novel family of triazine-based inhibitors of sorbitol dehydrogenase with oral activity: 1-\{4-[3R, 5S-dimethyl-4-(4methyl-[1, 3, 5] triazin-2-yl)-piperazin-1-yl]-[1, 3, 5] triazin-2-yl\}-(R) ethanol. Biorg Med Chem 11(19):4179-4188

6. Henke BR, Consler TG, Go N, Hale RL, Hohman DR, Jones SA et al (2002) A new series of estrogen receptor modulators that display selectivity for estrogen receptor $\beta$. J Med Chem 45(25):5492-5505

7. Klenke B, Stewart M, Barrett MP, Brun R, Gilbert IH (2001) Synthesis and biological evaluation of s-triazine substituted polyamines as potential new anti-trypanosomal drugs. J Med Chem 44(21):3440-3452

8. D'Atri G, Gomarasca P, Resnati G, Tronconi G, Scolastico C, Sirtori CR (1984) Novel pyrimidine and 1, 3, 5-triazine hypolipemic agents. J Med Chem 27(12):1621-1629

9. Jensen NP, Ager AL, Bliss RA, Canfield CJ, Kotecka BM, Rieckmann KH et al (2001) Phenoxypropoxybiguanides, prodrugs of DHFR-inhibiting diaminotriazine antimalarials. J Med Chem 44(23):3925-3931

10. Agarwal A, Srivastava K, Puri S, Chauhan PM (2005) Syntheses of 2, 4 , 6-trisubstituted triazines as antimalarial agents. Biorg Med Chem Lett. 15(3):531-533

11. Srinivas K, Srinivas U, Rao VJ, Bhanuprakash K, Kishore KH, Murty U (2005) Synthesis and antibacterial activity of 2, 4, 6-tri substituted s-triazines. Biorg Med Chem Lett 15(4):1121-1123

12. McKay GA, Reddy R, Arhin F, Belley A, Lehoux D, Moeck G et al (2006) Triaminotriazine DNA helicase inhibitors with antibacterial activity. Biorg Med Chem Lett. 16(5):1286-1290

13. Ghaib A, Ménager $S$, Vérité $P$, Lafont $O$ (2002) Synthesis of variously 9 , 9-dialkylated octahydropyrimido [3, 4-a]-s-triazines with potential antifungal activity. II Farmaco. 57(2):109-116
14. Lübbers T, Angehrn P, Gmünder H, Herzig S, Kulhanek J (2000) Design, synthesis, and structure-activity relationship studies of ATP analogues as DNA gyrase inhibitors. Biorg Med Chem Lett. 10(8):821-826

15. Lebreton S, Newcombe N, Bradley M (2003) Antibacterial single-bead screening. Tetrahedron 59(51):10213-10222

16. Sunduru N, Sharma M, Srivastava K, Rajakumar S, Puri S, Saxena J et al (2009) Synthesis of oxalamide and triazine derivatives as a novel class of hybrid 4-aminoquinoline with potent antiplasmodial activity. Biorg Med Chem. 17(17):6451-6462

17. Silen JL, Lu AT, Solas DW, Gore MA, Maclean D, Shah NH et al (1998) Screening for novel antimicrobials from encoded combinatorial libraries by using a two-dimensional agar format. Antimicrob Agents Chemother 42(6):1447-1453

18. Zhou C, Min J, Liu Z, Young A, Deshazer H, Gao T et al (2008) Synthesis and biological evaluation of novel 1, 3, 5-triazine derivatives as antimicrobial agents. Biorg Med Chem Lett. 18(4):1308-1311

19. Koc ZE, Bingol H, Saf AO, Torlak E, Coskun A (2010) Synthesis of novel tripodal-benzimidazole from 2, 4, 6-tris ( $\mathrm{p}$-formylphenoxy)-1, 3, 5-triazine: structural, electrochemical and antimicrobial studies. J Hazard Mater 183(1):251-255

20. Desai N, Makwana AH, Rajpara K (2016) Synthesis and study of 1, 3, 5-triazine based thiazole derivatives as antimicrobial agents. J Saudi Chem Soc 20:S334-S341

21. Vembu S, Pazhamalai S, Gopalakrishnan M (2015) Potential antibacterial activity of triazine dendrimer: synthesis and controllable drug release properties. Biorg Med Chem 23(15):4561-4566

22. Patel $A B$, Chikhalia KH, Kumari P (2014) An efficient synthesis of new thiazolidin-4-one fused s-triazines as potential antimicrobial and anticancer agents. J Saudi Chem Soc. 18(5):646-656

23. Shanmugakala R, Tharmaraj P, Sheela C (2014) Synthesis and spectral studies on metal complexes of s-triazine based ligand and non linear optical properties. J Mol Struct 1076:606-613

24. Shanmugam M, Narayanan K, Chidambaranathan V, Kabilan S (2013) Synthesis, spectral characterization and antimicrobial studies of novel s-triazine derivatives. Spectrochim Acta Mol Biomol Spectrosc. 105:383-390

25. Avupati VR, Yejella RP, Parala VR, Killari KN, Papasani VMR, Cheepurupalli P et al (2013) Synthesis, characterization and in vitro biological evaluation of some novel 1, 3, 5-triazine-Schiff base conjugates as potential antimycobacterial agents. Biorg Med Chem Lett. 23(21):5968-5970

26. Foster BJ, Harding BJ, Leyland-Jones B, Hoth D (1986) Hexamethylmelamine: a critical review of an active drug. Cancer Treat Rev 13(4):197-217

27. Ono M, Kawahara N, Goto D, Wakabayashi Y, Ushiro S, Yoshida S et al (1996) Inhibition of tumor growth and neovascularization by an antigastric ulcer agent, irsogladine. Cancer Res 56(7):1512-1516

28. Tranchand B, Catimel G, Lucas C, Sarkany M, Bastian G, Evene E et al (1998) Phase I clinical and pharmacokinetic study of S9788, a new multidrug-resistance reversal agent given alone and in combination with doxorubicin to patients with advanced solid tumors. Cancer Chemother Pharmacol 41(4):281-291

29. Maeda M, Ligo M, Tsuda H, Fujita H, Yonemura Y, Nakagawa K et al (2000) Antimetastatic and antitumor effects of 2, 4-diamino-6-(pyridine-4-yl)-1, 3, 5-triazine (4PyDAT) on the high lung metastatic colon 26 tumor in mice. Anticancer Drug Des 15(3):217-223

30. Menicagli R, Samaritani S, Signore G, Vaglini F, Dalla Via L (2004) In vitro cytotoxic activities of 2-alkyl-4, 6-diheteroalkyl-1, 3, 5-triazines: new molecules in anticancer research. J Med Chem 47(19):4649-4652

31. Baindur N, Chadha N, Brandt BM, Asgari D, Patch RJ, Schalk-HiHi C et al (2005) 2-Hydroxy-4, 6-diamino-[1, 3, 5] triazines: a novel class of VEGF-R2 (KDR) tyrosine kinase inhibitors. J Med Chem 48(6):1717-1720

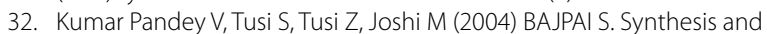
biological activity of substituted 2, 4, 6-s-triazines. Acta Pharm. 54(1):1-12

33. Gavade SN, Markad VL, Kodam KM, Shingare MS, Mane DV (2012) Synthesis and biological evaluation of novel 2, 4, 6-triazine derivatives as antimicrobial agents. Biorg Med Chem Lett 22(15):5075-5077

34. Chu DT, Plattner JJ, Katz L (1996) New directions in antibacterial research. J Med Chem 39(20):3853-3874

35. Beović B (2006) The issue of antimicrobial resistance in human medicine. Int J Food Microbiol 112(3):280-287

36. Finch $R$, Hunter $P$ (2006) Antibiotic resistance-action to promote new technologies: report of an EU Intergovernmental Conference held in 
Birmingham, UK, 12-13 December 2005. J Antimicrob Chemother 58(suppl 1):i3-i22

37. Suree N, Jung M, Clubb R (2007) Recent advances towards new antiinfective agents that inhibit cell surface protein anchoring in Staphylococcus aureus and other gram-positive pathogens. Mini Rev Med Chem 7(10):991-1000

38. Clark AM (1996) Natural products as a resource for new drugs. Pharm Res 13(8):1133-1141

39. Cowan MM (1999) Plant products as antimicrobial agents. Clin Microbiol Rev 12(4):564-582

40. Rex JH, Walsh TJ, Sobel JD, Filler SG, Pappas PG, Dismukes WE et al (2000) Practice guidelines for the treatment of candidiasis. Clin Infect Dis 30(4):662-678

41. Sanglard D, Odds FC (2002) Resistance of Candida species to antifungal agents: molecular mechanisms and clinical consequences. Lancet Infect Dis. 2(2):73-85

42. Buzzini P, Arapitsas P, Goretti M, Branda E, Turchetti B, Pinelli P et al (2008) Antimicrobial and antiviral activity of hydrolysable tannins. Mini Rev Med Chem 8(12):1179-1187

43. Funfuenha W, Phakhodee W, Pattarawarapan M (2014) Facile and efficient synthesis of C 2-symmetrical 1, 3, 5-triazine polycarboxylate ligands under microwave irradiation. Tetrahedron 70(35):5415-5419
44. Pavia DL, Lampman GM, Kriz GS, Vyvyan JA (2008) Introduction to spectroscopy. Cengage Learning, London

45. Henderson B, Dale M, Foreman J, Fan T (1994) Textbook of Immunopharmacology. Blackwell Scientific Publications, London, p 16

46. Mosmann T (1983) Rapid colorimetric assay for cellular growth and survival: application to proliferation and cytotoxicity assays. J Immunol Methods 65(1-2):55-63

47. Ragno R, Simeoni S, Castellano S, Vicidomini C, Mai A, Caroli A et al (2007) Small molecule inhibitors of histone arginine methyltransferases: homology modeling, molecular docking, binding mode analysis, and biological evaluations. J Med Chem 50(6):1241-1253

48. Renfrew AHM, Taylor JA, Whitmore JMJ, Williams A (1994) Nucleophilic aromatic substitution in heterocycles: alcoholysis and hydrolysis of 2-anilino-4,6-dichloro-1,3,5-triazines. J Chem Soc, Perkin Trans. 12:2389-2393

49. Maga G, Falchi F, Radi M, Botta L, Casaluce G, Bernardini M et al (2011) Toward the discovery of novel anti-HIV drugs. Second-generation inhibitors of the cellular ATPase DDX3 with improved anti-HIV activity: synthesis, structure-activity relationship analysis, cytotoxicity studies, and target validation. Chem Med Chem 6(8):1371-1389

50. Coxall RA, Harris SG, Henderson DK, Parsons S, Tasker PA, Winpenny RE (2000) Inter-ligand reactions: in situ formation of new polydentate ligands. J Chem Soc, Dalton Trans 14:2349-2356

\section{Submit your manuscript to a SpringerOpen ${ }^{\circ}$ journal and benefit from:}

- Convenient online submission

- Rigorous peer review

- Immediate publication on acceptance

- Open access: articles freely available online

- High visibility within the field

- Retaining the copyright to your article 